\title{
HOMOGENIZATION OF EVOLUTION PROBLEMS FOR A COMPOSITE MEDIUM WITH VERY SMALL AND HEAVY INCLUSIONS
}

\author{
Michel Bellieud ${ }^{1}$
}

\begin{abstract}
We study the homogenization of parabolic or hyperbolic equations like

$$
\rho_{\varepsilon} \frac{\partial^{n} u_{\varepsilon}}{\partial t^{n}}-\operatorname{div}\left(a_{\varepsilon} \nabla u_{\varepsilon}\right)=f \quad \text { in } \quad \Omega \times T+\text { boundary conditions, } \quad n \in\{1,2\},
$$

when the coefficients $\rho_{\varepsilon}, a_{\varepsilon}$ (defined in $\Omega$ ) take possibly high values on a $\varepsilon$-periodic set of grain-like inclusions of vanishing measure. Memory effects arise in the limit problem.
\end{abstract}

Mathematics Subject Classification. 35K05, 35L05, 73B27.

Received April 2, 2004. Revised September 13, 2004.

\section{INTRODUCTION}

We are concerned with the homogenization of parabolic or hyperbolic boundary-value problems of the type

$$
\left\{\begin{array}{l}
\rho_{\varepsilon} \frac{\partial^{n} u_{\varepsilon}}{\partial t^{n}}-\operatorname{div}\left(a_{\varepsilon} \nabla u_{\varepsilon}\right)=f \quad \text { in } \Omega \times T, \quad n \in\{1,2\} \\
+ \text { boundary conditions }
\end{array}\right.
$$

when the coefficients $\rho_{\varepsilon}, a_{\varepsilon}$ do not satisfy the assumptions of uniform ellipticity and boundedness like $0<\alpha<$ $a_{\varepsilon}(x), \rho_{\varepsilon}(x)<\beta<+\infty$ which guarantee a classical asymptotic behaviour. It is well known that in this case, homogenization may lead to unusual models such as non-local ones [7,9]. As far as scalar elliptic equations are concerned, it actually seems that the effective equation obtained by the homogenization of grain-like inclusions should be of local type (see Rem. $2.2(\mathrm{v})$ ). With regard to evolution equations on the contrary, we bring to the fore in this paper the possible presence of memory terms in the limit equation, when inclusions of high mass density and vanishing measure are considered.

In Section 2 we fix the notations and state the result (Th. 2.1). The proof, based on the argument developed in [1], is situated in Section 4. Section 3 is devoted to a priori estimates.

\footnotetext{
Keywords and phrases. Homogenization, memory effects, grain-like inclusions.

1 Département de Mathématiques, Université de Perpignan, 52 av. de Villeneuve, 66100 Perpignan, France; bellieud@univ-perp.fr
} 


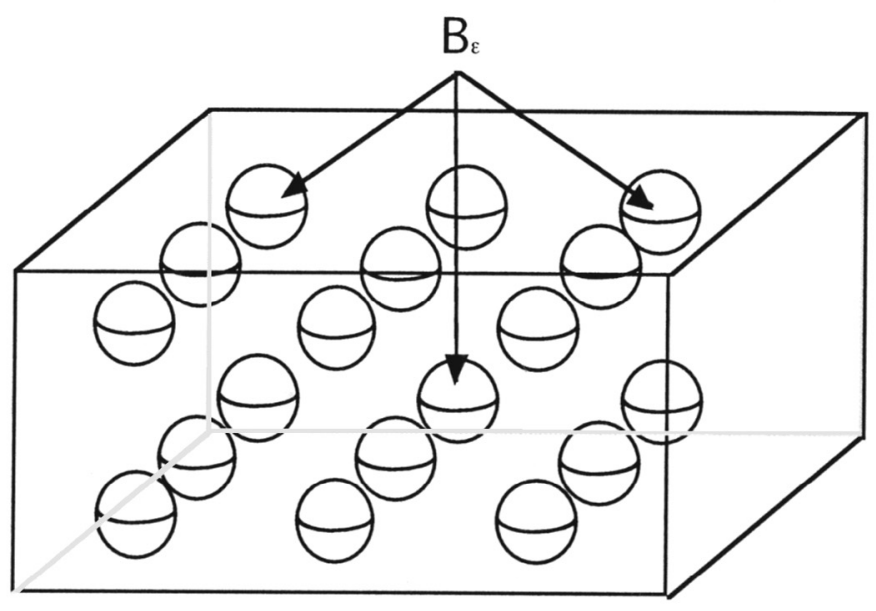

$\Omega$
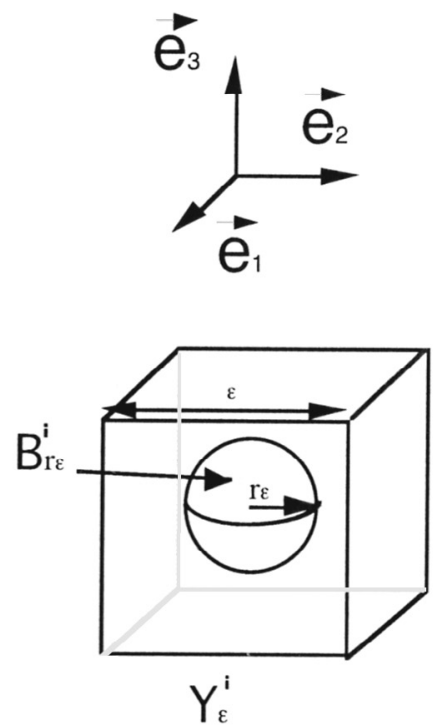

Figure 1. The composite medium.

\section{Notations AND MAIN RESUlts}

Let $\Omega$ be a bounded open domain of $\mathbb{R}^{3}$ with smooth boundary. Given a sequence of positive real numbers $\left(r_{\varepsilon}\right)$, the $\varepsilon$-periodic distribution $B_{\varepsilon}$ of balls of radius $r_{\varepsilon}$ is described as follows (see Fig. 1): we introduce

$$
\begin{aligned}
Y:=\left(-\frac{1}{2}, \frac{1}{2}\right)^{3} ; \quad B_{r}:=\left\{x \in \mathbb{R}^{3}, \sqrt{x_{1}^{2}+x_{2}^{2}+x_{3}^{2}}<r\right\} ; \\
Y_{\varepsilon}^{i}:=\varepsilon(\{i\}+Y) ; \quad B_{r}^{i}:=\varepsilon i+B_{r} ; \quad I_{\varepsilon}:=\left\{i \in Z^{3}, Y_{\varepsilon}^{i} \subset \Omega\right\},
\end{aligned}
$$

and set

$$
B_{\varepsilon}:=\bigcup_{i \in I_{\varepsilon}} B_{r_{\varepsilon}}^{i} .
$$

Our aim is to study the asymptotic behaviour of the sequence of evolution problems

$$
\rho_{\varepsilon} \frac{\partial^{n} u_{\varepsilon}}{\partial t^{n}}-\operatorname{div}\left(a_{\varepsilon} \nabla u_{\varepsilon}\right)=f \quad \text { in } \Omega \times T, \quad u_{\varepsilon} \in \mathcal{D}_{n}
$$

with

$$
n \in\{1,2\}, f \in L^{2}(\Omega \times T), U_{0} \in C_{0}^{1}(\bar{\Omega}), V_{0} \in C(\bar{\Omega}), 0<T<+\infty,
$$

and

$$
\begin{aligned}
& \mathcal{D}_{1}:=\left\{u \in L^{2}\left(0, T ; H_{0}^{1}(\Omega)\right) \cap C\left([0, T], L^{2}(\Omega)\right), u(0)=U_{0} \quad \text { in } \Omega\right\}, \\
& \mathcal{D}_{2}:=\left\{u \in C\left([0, T] ; H_{0}^{1}(\Omega)\right) \cap C^{1}\left([0, T] ; L^{2}(\Omega)\right), u(0)=U_{0}, \frac{\partial u}{\partial t}(0)=V_{0} \quad \text { in } \Omega\right\} .
\end{aligned}
$$

The sequences $\rho_{\varepsilon}, a_{\varepsilon}$ are defined by

$$
\begin{array}{ll}
\rho_{\varepsilon}(x)=\rho_{1 \varepsilon} & \text { if } x \in B_{\varepsilon}, \quad \rho_{\varepsilon}(x)=\rho_{0}>0 \text { if } x \in \Omega \backslash B_{\varepsilon}, \\
a_{\varepsilon}(x)=a_{1 \varepsilon} & \text { if } x \in B_{\varepsilon}, \quad a_{\varepsilon}(x)=1 \text { if } x \in \Omega \backslash B_{\varepsilon}
\end{array}
$$


where $\left(\rho_{1 \varepsilon}\right)$ and $\left(a_{1 \varepsilon}\right)$ are two sequences of positive real numbers such that

$$
\begin{array}{ll}
\rho_{1 \varepsilon}, a_{\varepsilon}>c>0, & \lim _{\varepsilon \rightarrow 0} \frac{4 \pi}{3} \frac{r_{\varepsilon}^{3}}{\varepsilon^{3}} \rho_{1 \varepsilon}=\bar{\rho}_{1}, \quad \bar{\rho}_{1} \in[0,+\infty[, \\
\lim _{\varepsilon \rightarrow 0} a_{1 \varepsilon}=+\infty, & \sup _{\varepsilon>0} \int_{\Omega} a_{\varepsilon}\left|\nabla U_{0}\right|^{2} \mathrm{~d} x<+\infty .
\end{array}
$$

The function $\rho_{\varepsilon}$ is allowed to take very high values on the subset $B_{\varepsilon}$ of disconnected balls, while at the same time the measure of $B_{\varepsilon}$ tends to 0 . More precisely we assume

$$
0<r_{\varepsilon} \ll \varepsilon .
$$

The limit problem depends on $\bar{\rho}_{1}$ defined by (2.7), and on the parameter

$$
\gamma:=\lim _{\varepsilon \rightarrow 0} \frac{r_{\varepsilon}}{\varepsilon^{3}} \in[0,+\infty] .
$$

It is expressed in terms of the limit $u$ of the sequence $u_{\varepsilon}$ of solutions of (2.3) and the limit $v$ of the sequence $\left(v_{\varepsilon}\right)$ defined by

$$
v_{\varepsilon}:=\frac{|\Omega|}{\left|B_{\varepsilon}\right|} u_{\varepsilon} 1_{B_{\varepsilon} \times(0, T)},
$$

which describes the average behaviour of the restriction of $u_{\varepsilon}$ to the subset of balls $B_{\varepsilon}$. The effective boundary conditions are given by $(u, v) \in \mathcal{D}_{n}^{\text {eff }}$, where

$$
\mathcal{D}_{n}^{\mathrm{eff}}:=\left\{(u, v) \in\left(L^{2}\left(0, T ; H_{0}^{1}(\Omega)\right) \times L^{2}\left(0, T ; L^{2}(\Omega)\right)\right) \cap\left(\mathcal{C}_{n}\left(\rho_{0}\right) \times \mathcal{C}_{n}\left(\bar{\rho}_{1}\right)\right)\right\},
$$

with

$$
\begin{aligned}
& \mathcal{C}_{n}(0):=L^{2}\left(0, T ; L^{2}(\Omega)\right), \\
& \mathcal{C}_{n}(r):=\left\{g \in C^{n-1}\left([0, T] ; L^{2}(\Omega)\right), \begin{array}{c}
g(0)=U_{0} \text { if } n=1, \\
g(0)=U_{0} \text { and } \frac{\partial g}{\partial t}(0)=V_{0} \text { if } n=2
\end{array}\right\} \quad \text { if } r>0 .
\end{aligned}
$$

Notice that the function $u$ always satisfies the initial condition, while $v$ only satisfies it if $\bar{\rho}_{1}>0$.

Theorem 2.1. Assume (2.6), (2.7) and (2.8), then consider $\gamma$ defined in (2.9):

(i) if $\gamma>0$, the sequence $\left(u_{\varepsilon}\right)$ of solutions of (2.3) converges weakly in $L^{2}\left(0, T ; H_{0}^{1}(\Omega)\right.$ ) (resp. star-weakly in $L^{\infty}\left(0, T ; H_{0}^{1}(\Omega)\right)$ if $\left.n=2\right)$ to $u$ and the sequence $v_{\varepsilon}$ defined by (2.10) converges star-weakly in $\mathcal{M}(\overline{\Omega \times T})$ (resp. star-weakly in $L^{\infty}(0, T ; \mathcal{M}(\bar{\Omega}))$ if $\left.n=2\right)$ to a function $v$, where $(u, v)$ is the unique solution in $\mathcal{D}_{n}^{\text {eff }}$ of

$$
\begin{aligned}
& \begin{cases}\rho_{0} \frac{\partial^{n} u}{\partial t^{n}}-\Delta u+4 \pi \gamma(u-v)=f \quad \text { in } \quad \Omega \times T, & \text { if } \quad 0<\gamma<+\infty, \\
\bar{\rho}_{1} \frac{\partial^{n} v}{\partial t^{n}}+4 \pi \gamma(v-u)=0 \quad \text { in } \quad \Omega \times T, & \text { if } \gamma=+\infty .\end{cases} \\
& v=u ; \quad\left(\rho_{0}+\bar{\rho}_{1}\right) \frac{\partial^{n} u}{\partial t^{n}}-\Delta u=f \quad \text { in } \quad \Omega \times T,
\end{aligned}
$$

(ii) If $\gamma=0$, the sequence $\left(u_{\varepsilon}\right)$ of solutions of (2.3) converges weakly in $L^{2}\left(0, T ; H_{0}^{1}(\Omega)\right)$ (resp. star-weakly in $L^{\infty}\left(0, T ; H_{0}^{1}(\Omega)\right)$ if $\left.n=2\right)$ to the solution of

$$
\rho_{0} \frac{\partial^{n} u}{\partial t^{n}}-\Delta u=f \quad \text { in } \quad \Omega \times T .
$$




\section{Remark 2.2.}

i) The average value of the coefficient $a_{\varepsilon}(x)$ on the set $B_{\varepsilon}$ has no influence upon the limit problem, unlike in the case where $B_{\varepsilon}$ is assumed to be a periodic distribution of fibers (see [1,2]).

ii) If $\gamma=0$ and $\bar{\rho}_{1}>0$ the sequence $\left(v_{\varepsilon}\right)$ converges star-weakly in $\mathcal{M}(\overline{\Omega \times T})$ to the solution $v$ of the second line of (2.13) which satisfies the initial conditions given by (2.11), namely $v=U_{0}$ if $n=1$ and $v=U_{0}+V_{0} t$ if $n=2$ (this is proved at the end of Sect. 4). Of course, in this case the variables $u$ and $v$ are independent.

iii) The auxiliary variable $v$ can be expressed in terms of $u$ by solving the second equation in (2.13). Assuming $\bar{\rho}_{1}>0$ and setting $\omega^{2}=\frac{4 \pi \gamma}{\bar{\rho}_{1}}$, we obtain

$$
\begin{cases}v(x, t)=\int_{0}^{t} \omega^{2} \exp \left(\omega^{2}(\tau-t)\right) u(x, \tau) \mathrm{d} \tau+U_{0}(x) \exp \left(-\omega^{2} t\right), & \text { if } n=1 \\ v(x, t)=\int_{0}^{t} \omega \sin \omega(t-\tau) u(x, \tau) \mathrm{d} \tau+V_{0}(x) \frac{\sin \omega t}{\omega}+U_{0}(x) \cos \omega t, & \text { if } n=2\end{cases}
$$

yielding after substitution in the first equation

$$
\left\{\begin{aligned}
\rho_{0} \frac{\partial u}{\partial t}-\Delta u+\bar{\rho}_{1} \omega^{2}\left(u-\omega^{2} \int_{0}^{t} \exp \left(\omega^{2}(t-\tau)\right) u(\tau) \mathrm{d} \tau\right) & \\
=\rho_{0} f+\bar{\rho}_{1} \omega^{2} U_{0}(x) \exp \left(-\omega^{2} t\right), & \text { if } n=1 \\
\rho_{0} \frac{\partial^{2} u}{\partial t^{2}}-\Delta u+\bar{\rho}_{1} \omega^{2}\left(u-\omega \int_{0}^{t} \sin (\omega(t-\tau)) u(\tau) \mathrm{d} \tau\right) & \\
=\rho_{0} f+\bar{\rho}_{1} \omega\left(V_{0}(x) \sin (\omega t)+\omega U_{0}(x) \cos (\omega t)\right), & \text { if } n=2
\end{aligned}\right.
$$

bringing to the fore the presence of memory terms in the limit problem.

iv) The asymptotic behaviour of

$$
\rho_{\varepsilon} \frac{\partial^{n} u_{\varepsilon}}{\partial t^{n}}-\operatorname{div}\left(a_{\varepsilon} \nabla u_{\varepsilon}\right)=\rho_{\varepsilon} f \quad \text { in } \Omega \times T, \quad u_{\varepsilon} \in \mathcal{D}_{n}
$$

can be studied in the same way, provided we assume

$$
\gamma>0, \quad f \in C(\overline{\Omega \times T})
$$

(if $\gamma=0$, the relative compactness of $\left(u_{\varepsilon}\right)$ may fail). The effective equations read

$$
\begin{aligned}
& (u, v) \in \mathcal{D}_{n}^{\mathrm{eff}}, \\
& \left\{\begin{array}{l}
\rho_{0} \frac{\partial^{n} u}{\partial t^{n}}-\Delta u+4 \pi \gamma(u-v)=\rho_{0} f \quad \text { in } \quad \Omega \times T, \\
\bar{\rho}_{1} \frac{\partial^{n} v}{\partial t^{n}}+4 \pi \gamma(v-u)=\bar{\rho}_{1} f \quad \text { in } \quad \Omega \times T,
\end{array}\right. \\
& v=u ; \quad\left(\rho_{0}+\bar{\rho}_{1}\right) \frac{\partial^{n} u}{\partial t^{n}}-\Delta u=\left(\rho_{0}+\bar{\rho}_{1}\right) f \quad \text { on } \quad \Omega \times T, \quad \text { if } \quad \gamma=+\infty
\end{aligned}
$$

v) The homogenization of the sequence of elliptic problems

$$
-\operatorname{div}\left(a_{\varepsilon} \nabla u_{\varepsilon}\right)=\rho_{\varepsilon}(x) f \quad \text { in } \quad \Omega, \quad u_{\varepsilon} \in H_{0}^{1}(\Omega),
$$


can be obtained by the same method under the asumptions (2.6), (2.7) (of course, with no assumption related to the initial condition), (2.8), (2.15). The effective equation satisfied by the weak limit $u$ in $H_{0}^{1}(\Omega)$ of the sequence $\left(u_{\varepsilon}\right)$ of solutions of (2.17), deduced from (2.13) by substituting 0 for the time derivatives, reads (see a sketch of the proof at the end of Sect. 4)

$$
-\Delta u=\left(\rho_{0}+\bar{\rho}_{1}\right) f \quad \text { in } \Omega, \quad u \in H_{0}^{1}(\Omega) .
$$

This colour of simplicity covers the interesting behaviour of the sequence $\left(v_{\varepsilon}\right)$, which converges star-weakly in the sense of measures to the function $v \in L^{2}(\Omega)$ such that $(u, v)$ is the solution in $H_{0}^{1}(\Omega) \times L^{2}(\Omega)$ of

$$
\begin{aligned}
& \left\{\begin{array}{l}
-\Delta u+4 \pi \gamma(u-v)=\rho_{0} f \quad \text { in } \quad \Omega, \\
4 \pi \gamma(v-u)=\bar{\rho}_{1} f \quad \text { in } \Omega,
\end{array} \quad \text { if } \quad 0<\gamma<+\infty,\right. \\
& v=u ; \quad-\Delta u=\left(\rho_{0}+\bar{\rho}_{1}\right) f \quad \text { on } \quad \Omega, \quad \text { if } \quad \gamma=+\infty \text {. }
\end{aligned}
$$

The couple of equations (2.19) is the Euler-Lagrange system associated with

$$
\min _{(u, v) \in\left(L^{2}(\Omega)\right)^{2}} \Phi(u, v)-\int_{\Omega} \rho_{0} f u \mathrm{~d} x-\int_{\Omega} \bar{\rho}_{1} f v \mathrm{~d} x,
$$

where the functional $\Phi$, defined on $\left(L^{2}(\Omega)\right)^{2}$ by

$$
\Phi(u, v):=\frac{1}{2} \int_{\Omega}|\nabla u|^{2} \mathrm{~d} x+\frac{1}{2} \int_{\Omega} 4 \pi \gamma(v-u)^{2} \mathrm{~d} x \quad \text { if } \quad u \in H_{0}^{1}(\Omega), \quad \Phi(u, v):=+\infty \quad \text { otherwise, }
$$

describes the energy associated with the couple $(u, v)$. By fitting the argument developed in [2], we can prove that the sequence of functionals defined on $L^{2}(\Omega)$ by $F_{\varepsilon}(u):=\frac{1}{2} \int_{\Omega} a_{\varepsilon}|\nabla u|^{2} \mathrm{~d} x-\int_{\Omega} \rho_{\varepsilon}(x) f . u \mathrm{~d} x$, if $u \in H_{0}^{1}(\Omega)$, $F_{\varepsilon}(u):=+\infty$ otherwise, $\Gamma$-converges (see $[6]$ ) strongly in $L^{2}(\Omega)$ to the functional

$$
\begin{aligned}
F(u) & :=\min _{v \in L^{2}\left(\Omega, \mathbb{R}^{3}\right)} \Phi(u, v)-\int_{\Omega} \rho_{0} f u \mathrm{~d} x-\int_{\Omega} \bar{\rho}_{1} f v \mathrm{~d} x \\
& =\frac{1}{2} \int_{\Omega}|\nabla u|^{2} \mathrm{~d} x-\int_{\Omega}\left(\rho_{0}+\bar{\rho}_{1}\right) f u \mathrm{~d} x-\frac{1}{8 \pi \gamma} \int_{\Omega}\left(\bar{\rho}_{1} f\right)^{2} \mathrm{~d} x .
\end{aligned}
$$

Classically, the solutions $u_{\varepsilon}$ of $(2.17)$, which minimize $F_{\varepsilon}$, converge to the solution of $\min _{u} F(u)$ equivalent to $(2.18)$.

vi) The results of $[1,2]$ corresponding to fiber structures have recently been extended to the framework of linear elasticity $($ see $[3,4])$. It is likely possible to do the same for grain-like inclusions, although there occurs an additional difficulty relating to the calculation of the exact solution of a three dimensional elasticity elementary problem (corresponding to (4.5)).

vii) Inclusions of high mass density with a radius $r_{\varepsilon}$ of the same order of magnitude as the period $\varepsilon$ have been considered in [10].

\section{A PRIORI ESTIMATES}

In the sequel, the letter "C" represents a suitable positive constant, independent of $\varepsilon$, which may vary from line to line. We introduce the following measures on $\Omega$ defined by

$$
m_{\varepsilon}:=\frac{3}{4 \pi} \frac{\varepsilon^{3}}{r_{\varepsilon}^{3}} \mathcal{L}^{3}\left\lfloor_ { B _ { \varepsilon } } \left(=\frac{\left|Y_{\varepsilon}^{i}\right|}{\left|B_{\varepsilon}^{i}\right|} \mathcal{L}^{3}\left\lfloor_{B_{\varepsilon}}\right) .\right.\right.
$$


We have $m_{\varepsilon}(\Omega)=\left|\cup_{i \in I_{\varepsilon}} Y_{\varepsilon}^{i}\right| \leq|\Omega|$, hence the sequence $\left(m_{\varepsilon}\right)$ is bounded in $\mathcal{M}(\bar{\Omega})$. Fixing $\varphi \in \mathcal{D}(\Omega)$ and setting

$$
\varphi_{\varepsilon}(x):=\sum_{i \in I_{\varepsilon}}\left(f_{Y_{\varepsilon}^{i}} \varphi(s) \mathrm{ds}\right) 1_{Y_{\varepsilon}^{i}}(x)
$$

(here the usual notation $f_{E} f \mathrm{~d} \nu=\frac{1}{\nu(E)} \int_{E} f \mathrm{~d} \nu$ is employed), noticing that

$$
\int \varphi_{\varepsilon} \mathrm{d} m_{\varepsilon}=\sum_{i \in I_{\varepsilon}} \frac{\left|Y_{\varepsilon}^{i}\right|}{\left|B_{\varepsilon}^{i}\right|} \int_{B_{\varepsilon}^{i}} \varphi_{\varepsilon} \mathrm{d} x=\int_{\cup_{i \in I_{\varepsilon}} Y_{\varepsilon}^{i}} \varphi \mathrm{d} x
$$

and that the inequality

$$
\left\|\varphi-\varphi_{\varepsilon}\right\|_{L^{\infty}(\Omega)} \leq C \varepsilon
$$

holds as soon as the support of $\varphi$ is included in $\cup_{i \in I_{\varepsilon}} Y_{\varepsilon}^{i}$ (i.e. for $\varepsilon<\frac{1}{\sqrt{3}} \operatorname{dist}(\partial \Omega$, Support $\varphi)$ ), we deduce

$$
\lim _{\varepsilon \rightarrow 0} \int \varphi \mathrm{d} m_{\varepsilon}=\lim _{\varepsilon \rightarrow 0} \int \varphi_{\varepsilon} \mathrm{d} m_{\varepsilon}=\lim _{\varepsilon \rightarrow 0} \int_{\cup_{i \in I_{\varepsilon}} Y_{\varepsilon}^{i}} \varphi \mathrm{d} x=\int_{\Omega} \varphi \mathrm{d} x
$$

and

$$
m_{\varepsilon} \stackrel{\star}{\lessgtr} \mathcal{L}^{3}\lfloor\Omega, \quad \text { star-weakly in } \mathcal{M}(\bar{\Omega}) .
$$

Let us fix a sequence of positive real numbers $\left(R_{\varepsilon}\right)$ such that

$$
r_{\varepsilon} \ll R_{\varepsilon} \ll \varepsilon \quad \text { and } \quad \varepsilon^{3} \ll R_{\varepsilon} .
$$

For a given sequence $\left(u_{\varepsilon}\right)$ in $H_{0}^{1}(\Omega)$, we introduce the following functions

$$
\begin{aligned}
& \tilde{u}_{\varepsilon}(x):=\sum_{i \in I_{\varepsilon}}\left(f_{\partial B_{R_{\varepsilon}}^{i}} u_{\varepsilon}(s) \mathrm{d} \mathcal{H}^{2}(s)\right) 1_{Y_{\varepsilon}^{i}}(x), \\
& \tilde{v}_{\varepsilon}(x):=\sum_{i \in I_{\varepsilon}}\left(f_{\partial B_{r_{\varepsilon}}^{i}} u_{\varepsilon}(s) \mathrm{d} \mathcal{H}^{2}(s)\right) 1_{Y_{\varepsilon}^{i}}(x),
\end{aligned}
$$

where $B_{R_{\varepsilon}}^{i}, B_{r_{\varepsilon}}^{i}, Y_{\varepsilon}^{i}, I_{\varepsilon}$ are defined by (2.1). Their asymptotic behaviour is characterized by

Lemma 3.1. Let $u_{\varepsilon}$ be a sequence in $H_{0}^{1}(\Omega)$ and $m_{\varepsilon},\left(\tilde{u}_{\varepsilon}\right),\left(\tilde{v}_{\varepsilon}\right)$ be defined by (3.1), (3.4). Then the following estimates hold:

$$
\begin{aligned}
& \int_{\Omega}\left|u_{\varepsilon}-\tilde{u}_{\varepsilon}\right|^{2} \mathrm{~d} x \leq C\left(\frac{\varepsilon^{3}}{R_{\varepsilon}}+\varepsilon^{\frac{2}{3}}\right) \int_{\Omega}\left|\nabla u_{\varepsilon}\right|^{2} \mathrm{~d} x, \\
& \int_{B_{\varepsilon}}\left|u_{\varepsilon}-\tilde{v}_{\varepsilon}\right|^{2} \mathrm{~d} x \leq C r_{\varepsilon}^{2} \int_{B_{\varepsilon}}\left|\nabla u_{\varepsilon}\right|^{2} \mathrm{~d} x, \\
& \int_{\Omega}\left|\tilde{u}_{\varepsilon}-\tilde{v}_{\varepsilon}\right|^{2} \mathrm{~d} x \leq C \frac{\varepsilon^{3}}{r_{\varepsilon}} \int_{\Omega}\left|\nabla u_{\varepsilon}\right|^{2} \mathrm{~d} x, \\
& \int_{\Omega}\left|\tilde{u}_{\varepsilon}\right|^{2} \mathrm{~d} x=\int\left|\tilde{u}_{\varepsilon}\right|^{2} \mathrm{~d} m_{\varepsilon} \quad, \quad \int_{\Omega}\left|\tilde{v}_{\varepsilon}\right|^{2} \mathrm{~d} x=\int\left|\tilde{v}_{\varepsilon}\right|^{2} \mathrm{~d} m_{\varepsilon} .
\end{aligned}
$$


Proof. For a given couple $\left(r_{1}, r_{2}\right)$ of positive real numbers such that $r_{1}<r_{2}$, the elementary minimization problem

$$
\Gamma\left(r_{1}, r_{2}\right):=\min _{\varphi \in H^{1}\left(\left[r_{1}, r_{2}\right]\right)}\left\{\int_{r_{1}}^{r_{2}}\left|\varphi^{\prime}(r)\right|^{2} r^{2} \mathrm{~d} r, \quad \varphi\left(r_{1}\right)=1, \quad \varphi\left(r_{2}\right)=0\right\},
$$

is achieved at $\varphi(r):=\frac{r_{1}}{r} \frac{r_{2}-r}{r_{2}-r_{1}}$, yielding

$$
\Gamma\left(r_{1}, r_{2}\right)=\frac{r_{1} r_{2}}{r_{2}-r_{1}}
$$

In accordance with (3.6) and Cauchy-Schwartz's inequality, for any $u \in H^{1}\left(B_{r_{2}} \backslash B_{r_{1}}\right)$ (see (2.1)) we have (in spherical coordinates)

$$
\begin{aligned}
\int_{B_{r_{2}} \backslash B_{r_{1}}}|\nabla u|^{2} \mathrm{~d} x & \geq \int_{\theta_{1}=0}^{\pi} \int_{\theta_{2}=0}^{2 \pi} \int_{r_{1}}^{r_{2}}\left|\frac{\partial u}{\partial r}\right|^{2} r^{2} \sin \theta_{1} \mathrm{~d} r \mathrm{~d} \theta_{1} \mathrm{~d} \theta_{2} \\
& \geq \Gamma\left(r_{1}, r_{2}\right) \int_{\theta_{1}=0}^{\pi} \int_{\theta_{2}=0}^{2 \pi}\left|u\left(r_{2}, \theta_{1}, \theta_{2}\right)-u\left(r_{1}, \theta_{1}, \theta_{2}\right)\right|^{2} \sin \theta_{1} \mathrm{~d} \theta_{1} \mathrm{~d} \theta_{2} \\
& \geq 4 \pi \Gamma\left(r_{1}, r_{2}\right)\left|\int_{\theta_{1}=0}^{\pi} \int_{\theta_{2}=0}^{2 \pi}\left(u\left(r_{2}, \theta_{1}, \theta_{2}\right)-u\left(r_{1}, \theta_{1}, \theta_{2}\right)\right) \frac{\sin \theta_{1}}{4 \pi} \mathrm{d} \theta_{1} \mathrm{~d} \theta_{2}\right|^{2} \\
& =4 \pi \Gamma\left(r_{1}, r_{2}\right)\left|f_{\partial B_{r_{2}}} u \mathrm{~d} \mathcal{H}^{2}-f_{\partial B_{r_{1}}} u \mathrm{~d} \mathcal{H}^{2}\right|^{2}
\end{aligned}
$$

Applying (3.8) on each subset $\left(B_{R_{\varepsilon}}^{i} \backslash B_{r_{\varepsilon}}^{i}\right)$, we deduce from (3.4), (3.7)

$$
\begin{aligned}
\int_{\Omega}\left|\tilde{u}_{\varepsilon}-\tilde{v}_{\varepsilon}\right|^{2} \mathrm{~d} x & =\sum_{i \in I_{\varepsilon}} \int_{Y_{\varepsilon}^{i}}\left|f_{\partial B_{R_{\varepsilon}}^{i}} u_{\varepsilon} \mathrm{d} \mathcal{H}^{2}-f_{\partial B_{r_{\varepsilon}}^{i}} u_{\varepsilon} \mathrm{d} \mathcal{H}^{2}\right|^{2} \mathrm{~d} x \\
& \leq \sum_{i \in I_{\varepsilon}} \int_{Y_{\varepsilon}^{i}}\left(\frac{1}{4 \pi \Gamma_{1}\left(R_{\varepsilon}, r_{\varepsilon}\right)} \int_{B_{R_{\varepsilon}}^{i} \backslash B_{r_{\varepsilon}}^{i}}\left|\nabla u_{\varepsilon}\right|^{2} \mathrm{~d} x\right) \mathrm{d} x \\
& \leq \sum_{i \in I_{\varepsilon}} \varepsilon^{3}\left(\frac{1}{4 \pi \Gamma_{1}\left(R_{\varepsilon}, r_{\varepsilon}\right)} \int_{B_{R_{\varepsilon}}^{i} \backslash B_{r_{\varepsilon}}^{i}}\left|\nabla u_{\varepsilon}\right|^{2} \mathrm{~d} x\right) \\
& \leq \frac{\varepsilon^{3}}{4 \pi \Gamma_{1}\left(R_{\varepsilon}, r_{\varepsilon}\right)} \int_{\Omega}\left|\nabla u_{\varepsilon}\right|^{2} \mathrm{~d} x=\frac{\varepsilon^{3}\left(R_{\varepsilon}-r_{\varepsilon}\right)}{4 \pi R_{\varepsilon} r_{\varepsilon}} \int_{\Omega}\left|\nabla u_{\varepsilon}\right|^{2} \mathrm{~d} x \\
& \leq \frac{\varepsilon^{3}}{4 \pi r_{\varepsilon}} \int_{\Omega}\left|\nabla u_{\varepsilon}\right|^{2} \mathrm{~d} x,
\end{aligned}
$$

and the third line of (3.5). Next we establish (see below) for $R>0$ and $\alpha \in] 0,1]$

$$
\int_{B_{R}}\left|u(x)-f_{\partial B_{\alpha R}} u(s) \mathrm{d} \mathcal{H}^{2}(s)\right|^{2} \mathrm{~d} x \leq C \frac{R^{2}}{\alpha} \int_{B_{R}}|\nabla u|^{2} \mathrm{~d} x, \quad \forall u \in H^{1}\left(B_{R}\right) .
$$

By applying (3.9) to $u_{\varepsilon}$ (possibly extended to $\mathbb{R}^{3}$ by setting $u_{\varepsilon}(x)=0$ if $x \in \mathbb{R}^{3} \backslash \Omega$ ) on each ball $B_{\frac{\sqrt{3}}{2} \varepsilon}^{i}$ (see (2.1)) with $R=\frac{\sqrt{3}}{2} \varepsilon$ and $\alpha=\frac{2}{\sqrt{3}} \frac{R_{\varepsilon}}{\varepsilon}$, noticing that $Y_{\varepsilon}^{i} \subset B_{\frac{\sqrt{3} \varepsilon}{2}}^{i}$ and $\sum_{i \in I_{\varepsilon}} 1_{B_{\frac{\sqrt{3}}{2} \varepsilon}^{i}} \leq 2$ on $\mathbb{R}^{3}$ (because the balls $B_{\frac{\sqrt{3}}{2} \varepsilon}^{i}$ 
intersect each other no more than two times), we infer

$$
\begin{aligned}
& \int_{\cup_{i \in I_{\varepsilon}} Y_{\varepsilon}^{i}}\left|u_{\varepsilon}-\tilde{u}_{\varepsilon}\right|^{2} \mathrm{~d} x \leq \sum_{i \in I_{\varepsilon}} \int_{Y_{\varepsilon}^{i}}\left|u_{\varepsilon}-\int_{\partial B_{R_{\varepsilon}}^{i}} u_{\varepsilon} \mathrm{d} \mathcal{H}^{2}\right|^{2} \mathrm{~d} x \\
& \leq \sum_{i \in I_{\varepsilon}} \int_{B_{\frac{\sqrt{3}}{2} \varepsilon}^{i}}\left|u_{\varepsilon}-f_{\partial B_{R_{\varepsilon}}^{i}} u_{\varepsilon} \mathrm{d} \mathcal{H}^{2}\right|^{2} \mathrm{~d} x \\
& \leq C \frac{\varepsilon^{3}}{R_{\varepsilon}} \sum_{i \in I_{\varepsilon}} \int_{B_{\frac{\sqrt{3}}{2} \varepsilon}}\left|\nabla u_{\varepsilon}\right|^{2} \mathrm{~d} x \leq C \frac{\varepsilon^{3}}{R_{\varepsilon}} \int_{\Omega}\left|\nabla u_{\varepsilon}\right|^{2} \mathrm{~d} x .
\end{aligned}
$$

On the other hand, setting $N_{\varepsilon}:=\Omega \backslash \cup_{i \in I_{\varepsilon}} Y_{\varepsilon}^{i}$ (notice that $N_{\varepsilon} \subset\{x \in \Omega$, $\operatorname{dist}(x, \partial \Omega) \leq \sqrt{3} \varepsilon\}$ hence there holds $\left|N_{\varepsilon}\right| \leq C \varepsilon$, because $\partial \Omega$ is smooth) by (3.4), Hölder's inequality and the continuous inbedding $H_{0}^{1}(\Omega) \subset L^{6}(\Omega)$ we have

$$
\int_{N_{\varepsilon}}\left|u_{\varepsilon}-\tilde{u}_{\varepsilon}\right|^{2} \mathrm{~d} x=\int_{N_{\varepsilon}}\left|u_{\varepsilon}\right|^{2} \mathrm{~d} x \leq|| u_{\varepsilon} \|_{L^{6}(\Omega)}^{2}\left|N_{\varepsilon}\right|^{\frac{2}{3}} \leq C \varepsilon^{\frac{2}{3}} \int_{\Omega}\left|\nabla u_{\varepsilon}\right|^{2} \mathrm{~d} x .
$$

We deduce the first line of (3.5) from (3.10) and (3.11). By repeating the argument on each ball $B_{r_{\varepsilon}}^{i}$ (with $R=r_{\varepsilon}, \alpha=1$ ), we get

$$
\begin{aligned}
\int_{B_{\varepsilon}}\left|u_{\varepsilon}-\tilde{v}_{\varepsilon}\right|^{2} \mathrm{~d} x & =\sum_{i \in I_{\varepsilon}} \int_{B_{r_{\varepsilon}}^{i}}\left|u_{\varepsilon}-f_{\partial B_{r_{\varepsilon}}^{i}} u_{\varepsilon} \mathrm{d} \mathcal{H}^{2}\right|^{2} \mathrm{~d} x \\
& \leq C r_{\varepsilon}^{2} \sum_{i \in I_{\varepsilon}} \int_{B_{r_{\varepsilon}}^{i}}\left|\nabla u_{\varepsilon}\right|^{2} \mathrm{~d} x=C r_{\varepsilon}^{2} \int_{B_{\varepsilon}}\left|\nabla u_{\varepsilon}\right|^{2} \mathrm{~d} x
\end{aligned}
$$

and the second line of (3.5). Finally by (3.1) and (3.4) there holds

$$
\begin{aligned}
\int\left|\tilde{u}_{\varepsilon}\right|^{2} \mathrm{~d} m_{\varepsilon} & =\sum_{i \in I_{\varepsilon}} \frac{\left|Y_{\varepsilon}^{i}\right|}{\left|B_{\varepsilon}^{i}\right|} \int_{B_{\varepsilon}^{i}}\left(f_{\partial B_{R_{\varepsilon}}^{i}} u_{\varepsilon}(s) \mathrm{d} \mathcal{H}^{2}(s)\right) \mathrm{d} x=\sum_{i \in I_{\varepsilon}} \int_{Y_{\varepsilon}^{i}}\left(f_{\partial B_{R_{\varepsilon}}^{i}} u_{\varepsilon}(s) \mathrm{d} \mathcal{H}^{2}(s)\right) \mathrm{d} x \\
& \left.=\int_{\Omega}\left|\tilde{u}_{\varepsilon}\right|^{2} \mathrm{~d} x, \quad \text { (resp. } \int\left|\tilde{v}_{\varepsilon}\right|^{2} \mathrm{~d} m_{\varepsilon}=\int_{\Omega}\left|\tilde{v}_{\varepsilon}\right|^{2} \mathrm{~d} x\right)
\end{aligned}
$$

yielding the fourth line of (3.5).

Proof of (3.9). We prove the inequality for $R=1$, the general case is inferred by making the change of variable $y=R x$. Noticing that by (3.7) and (3.8), for any $r \in] 0,1[$ we have

$$
\left|f_{\partial B_{r}} u \mathrm{~d} \mathcal{H}^{2}-f_{\partial B_{\alpha}} u \mathrm{~d} \mathcal{H}^{2}\right|^{2} \leq \frac{1}{4 \pi} \frac{|r-\alpha|}{r \alpha} \int_{B_{1}}|\nabla u|^{2} \mathrm{~d} x
$$


we deduce from Cauchy-Schwartz's inequality and (3.12) that

$$
\begin{aligned}
\left|f_{B_{1}} u \mathrm{~d} x-f_{\partial B_{\alpha}} u \mathrm{~d} \mathcal{H}^{2}\right|^{2} & =\left|\int_{0}^{1}\left(f_{\partial B_{r}} u \mathrm{~d} \mathcal{H}^{2}\right) 3 r^{2} \mathrm{~d} r-\int_{0}^{1}\left(f_{\partial B_{\alpha}} u \mathrm{~d} \mathcal{H}^{2}\right) 3 r^{2} \mathrm{~d} r\right|^{2} \\
& \leq \int_{0}^{1}\left|f_{\partial B_{r}} u \mathrm{~d} \mathcal{H}^{2}-f_{\partial B_{\alpha}} u \mathrm{~d} \mathcal{H}^{2}\right|^{2} 3 r^{2} \mathrm{~d} r \\
& \leq \int_{0}^{1} \frac{\left(\int_{B_{1}}|\nabla u|^{2} \mathrm{~d} x\right)}{4 \pi} \frac{|r-\alpha|}{r \alpha} 3 r^{2} \mathrm{~d} r \leq \frac{C}{\alpha} \int_{B_{1}}|\nabla u|^{2} \mathrm{~d} x
\end{aligned}
$$

then, from the Poincaré-Wirtinger's inequality $\int_{B_{1}}\left|u(x)-f_{B_{1}} u(s) \mathrm{d} s\right|^{2} \mathrm{~d} x \leq C \int_{B_{1}}|\nabla u|^{2} \mathrm{~d} x$, that

$$
\int_{B_{1}}\left|u-f_{\partial B_{\alpha}} u \mathrm{~d} \mathcal{H}^{2}\right|^{2} \mathrm{~d} x \leq \frac{C}{\alpha} \int_{B_{1}}|\nabla u|^{2} \mathrm{~d} x .
$$

We recall (see [2] Lem. A2, p. 431).

Lemma 3.2. Let $\nu_{\varepsilon}$ and $\nu$ be Radon measures on a compact $K \subset \mathbb{R}^{N}$ such that $\nu_{\varepsilon} \stackrel{\star}{*} \nu$. Let $\left(f_{\varepsilon}\right)$ a sequence of $\nu_{\varepsilon}$-measurable functions such that $\sup _{\varepsilon} \int\left|f_{\varepsilon}\right|^{2} \mathrm{~d} \nu_{\varepsilon}<+\infty$. Then the sequence $f_{\varepsilon} \nu_{\varepsilon}$ is sequentially relatively compact in the weak-star topology $\sigma(\mathcal{M}(K), C(K))$ and every cluster point $m$ is of the form $m=f \nu$ with $f \in L_{\nu}^{2}$. Moreover, if $f_{\varepsilon} \nu_{\varepsilon} \stackrel{\star}{\rightarrow} f \nu$, then $\liminf _{\varepsilon} \int\left|f_{\varepsilon}\right|^{2} \mathrm{~d} \nu_{\varepsilon} \geq \int|f|^{2} \mathrm{~d} \nu$.

The following proposition particularizes the asymptotic behaviour of the sequence $\left(u_{\varepsilon}\right)$ of solutions of $(2.3)$ and of the sequence $\left(v_{\varepsilon}\right)$ defined by $(2.10)$.

Proposition 3.3. The sequence $\left(u_{\varepsilon}\right)$ of solutions of (2.3) is bounded in $L^{2}\left(0, T ; H_{0}^{1}(\Omega)\right.$ ) (resp. bounded in $L^{\infty}\left(0, T ; H_{0}^{1}(\Omega)\right)$ if $\left.n=2\right)$. More precisely, the following estimates hold

$$
\begin{aligned}
& \int_{\Omega \times T}\left|\nabla u_{\varepsilon}\right|^{2} \mathrm{~d} x \mathrm{~d} t<C \quad\left(\text { resp. } \quad \int_{\Omega}\left|\nabla u_{\varepsilon}(x, \tau)\right|^{2} \mathrm{~d} x<C, \forall \tau \in[0, T], \quad \text { if } n=2\right), \\
& \int_{\Omega \times T} a_{\varepsilon}\left|\nabla u_{\varepsilon}\right|^{2} \mathrm{~d} x \mathrm{~d} t<C \quad\left(\text { resp. } \quad \int_{\Omega} a_{\varepsilon}\left|\nabla u_{\varepsilon}(x, \tau)\right|^{2} \mathrm{~d} x<C, \forall \tau \in[0, T], \quad \text { if } n=2\right),
\end{aligned}
$$

and up to a subsequence, there exists a function u such that

$$
\left.u_{\varepsilon} \rightarrow u \text { weakly in } L^{2}\left(0, T ; H_{0}^{1}(\Omega)\right) \text {, (resp. star-weakly in } L^{\infty}\left(0, T ; H_{0}^{1}(\Omega)\right) \text {, if } n=2\right) .
$$

Besides the sequence $\left(\tilde{u}_{\varepsilon}\right)$ defined by (3.4) satisfies

$$
\tilde{u}_{\varepsilon}-u_{\varepsilon} \rightarrow 0 \quad \text { strongly in } L^{2}(\Omega \times T)\left(\text { resp. in } L^{\infty}\left(0, T ; L^{2}(\Omega)\right) \text {, if } n=2\right) \text {. }
$$

Assume moreover that $\gamma>0$. Then the following estimate holds

$$
\begin{aligned}
& \int_{\Omega \times T}\left|u_{\varepsilon}\right|^{2} \mathrm{~d} m_{\varepsilon}(x) \mathrm{d} t \leq C, \quad \text { if } n=1, \\
& \text { (resp. } \left.\int_{\Omega}\left|u_{\varepsilon}(x, \tau)\right|^{2} \mathrm{~d} m_{\varepsilon}(x) \leq C, \quad \forall \tau \in[0, T], \quad \text { if } n=2\right) \text {, }
\end{aligned}
$$


and up to a subsequence, there exists $v \in L^{2}(\Omega \times T)$ such that

$$
\begin{aligned}
u_{\varepsilon} \mathrm{d} m_{\varepsilon} \mathrm{d} t \stackrel{\star}{\rightarrow} v \mathrm{~d} x \mathrm{~d} t \text { star-weakly in } \mathcal{M}(\overline{\Omega \times T}), \\
\left(\text { resp. star-weakly in } L^{\infty}(0, T, \mathcal{M}(\bar{\Omega})) \text { if } n=2\right) .
\end{aligned}
$$

In addition, the sequence $\left(\tilde{v}_{\varepsilon}\right)$ defined by (3.4) satisfies

$$
\tilde{v}_{\varepsilon} \rightarrow v \quad \text { weakly in } \quad L^{2}(\Omega \times T)\left(\text { resp. star-weakly in } L^{\infty}\left(0, T ; L^{2}(\Omega)\right) \text {, if } n=2\right) \text {. }
$$

Proof. Assuming first $n=1$, we multiply the equation (2.3) by $u_{\varepsilon}$ and integrate it over $\Omega \times(0, \tau)$ for a fixed real number $\tau \in[0, T]$. After integration by parts we obtain

$$
\frac{1}{2} \int_{\Omega} \rho_{\varepsilon}(x)\left|u_{\varepsilon}(x, \tau)\right|^{2} \mathrm{~d} x-\frac{1}{2} \int_{\Omega} \rho_{\varepsilon}(x)\left|U_{0}(x)\right|^{2} \mathrm{~d} x+\int_{0}^{\tau} \int_{\Omega} a_{\varepsilon}\left|\nabla u_{\varepsilon}\right|^{2} \mathrm{~d} x \mathrm{~d} t=\int_{0}^{\tau} \int_{\Omega} f u_{\varepsilon} \mathrm{d} x \mathrm{~d} t .
$$

Choosing $\tau=T$ in (3.20), thanks to the Poincaré's inequality and the fact that $\left(a_{\varepsilon}\right)$ can be bounded from below by a positive constant (see $(2.7))$, we infer

$$
\begin{aligned}
\left\|u_{\varepsilon}\right\|_{L^{2}(\Omega \times T)}^{2} & \leq C \int_{0}^{T} \int_{\Omega}\left|\nabla u_{\varepsilon}\right|^{2} \mathrm{~d} x \mathrm{~d} t \leq C \int_{0}^{T} \int_{\Omega} a_{\varepsilon}\left|\nabla u_{\varepsilon}\right|^{2} \mathrm{~d} x \mathrm{~d} t \\
& \leq C\|f\|_{L^{2}(\Omega \times T)}|| u_{\varepsilon} \|_{L^{2}(\Omega \times T)}+C \int_{\Omega} \rho_{\varepsilon}(x)\left|U_{0}(x)\right|^{2} \mathrm{~d} x,
\end{aligned}
$$

yielding the boundedness of $\left(u_{\varepsilon}\right)$ in $L^{2}\left(0, T ; H_{0}^{1}(\Omega)\right)$, (3.14) and (3.15) (the sequence of real numbers $\left(\frac{1}{2} \int_{\Omega} \rho_{\varepsilon}(x)\left|U_{0}(x)\right|^{2} \mathrm{~d} x\right)$ is bounded because by (2.7) the sequence of measures $\left(\rho_{\varepsilon}(x) \mathrm{d} x\right)$ is bounded in $\mathcal{M}(\bar{\Omega})$ and $U_{0}$ is continuous).

If $n=2$, by the standard regularity results (see [8] or [5], p. 222) we have

$$
u_{\varepsilon} \in C\left([0, T], H_{0}^{1}(\Omega)\right) \cap C^{1}\left([0, T], L^{2}(\Omega)\right), \quad \frac{\partial^{2} u_{\varepsilon}}{\partial t^{2}} \in L^{2}\left(0, T ; H^{-1}(\Omega)\right) .
$$

Fixing $t \in[0, T]$, multiplying the first line of (2.3) by $\frac{\partial u_{\varepsilon}}{\partial t}$ and integrating over $\Omega$, after integration by parts according to $(3.22)$ with respect to the space variables, we obtain

$$
\frac{\mathrm{d}}{\mathrm{d} t}\left(\frac{1}{2} \int_{\Omega} \rho_{\varepsilon}(x)\left|\frac{\partial u_{\varepsilon}}{\partial t}(x, t)\right|^{2} \mathrm{~d} x+\frac{1}{2} \int_{\Omega} a_{\varepsilon}\left|\nabla u_{\varepsilon}\right|^{2} \mathrm{~d} x\right)=\int_{\Omega} f(x, t) \frac{\partial u_{\varepsilon}(x, t)}{\partial t} \mathrm{~d} x .
$$

Fixing $\tau \in[0, T]$ and integrating (3.23) with respect to $t$ over $[0, \tau]$, thanks to the initial conditions given in $(2.3)$ we get

$$
\begin{aligned}
\frac{1}{2}\left(\int_{\Omega} \rho_{\varepsilon}(x)\left|\frac{\partial u_{\varepsilon}}{\partial t}(x, \tau)\right|^{2} \mathrm{~d} x+\right. & \left.\int_{\Omega} a_{\varepsilon}\left|\nabla u_{\varepsilon}\right|^{2} \mathrm{~d} x\right)= \\
& \frac{1}{2}\left(\int_{\Omega} \rho_{\varepsilon}(x)\left|V_{0}(x)\right|^{2} \mathrm{~d} x+\int_{\Omega} a_{\varepsilon}\left|\nabla U_{0}(x)\right|^{2} \mathrm{~d} x\right)+\int_{\Omega \times(0, \tau)} f(x, t) \frac{\partial u_{\varepsilon}(x, t)}{\partial t} \mathrm{~d} x \mathrm{~d} t .
\end{aligned}
$$


By (2.7), the sequence of real numbers $\left(\frac{1}{2} \int_{\Omega} \rho_{\varepsilon}(x)\left|V_{0}(x)\right|^{2} \mathrm{~d} x+\frac{1}{2} \int_{\Omega} a_{\varepsilon}\left|\nabla U_{0}(x)\right|^{2} \mathrm{~d} x\right)$ is bounded, hence we deduce from (3.24) that

$$
\frac{1}{2}\left(\int_{\Omega} \rho_{\varepsilon}(x)\left|\frac{\partial u_{\varepsilon}}{\partial t}(x, \tau)\right|^{2} \mathrm{~d} x+\int_{\Omega} a_{\varepsilon}\left|\nabla u_{\varepsilon}\right|^{2} \mathrm{~d} x\right) \leq C\left(1+\sqrt{\int_{\Omega \times T}\left|\frac{\partial u_{\varepsilon}}{\partial t}\right|^{2} \mathrm{~d} x \mathrm{~d} t}\right), \quad \forall \tau \in[0, T],
$$

and then, after integration over $(0, T)$ with respect to $\tau$,

$$
\frac{1}{2} \int_{\Omega \times T} \rho_{\varepsilon}(x)\left|\frac{\partial u_{\varepsilon}}{\partial t}\right|^{2} \mathrm{~d} x \mathrm{~d} t+\frac{1}{2} \int_{\Omega \times T} a_{\varepsilon}\left|\nabla u_{\varepsilon}\right|^{2} \mathrm{~d} x \mathrm{~d} t \leq C\left(1+\sqrt{\int_{\Omega \times T}\left|\frac{\partial u_{\varepsilon}}{\partial t}\right|^{2} \mathrm{~d} x \mathrm{~d} t}\right) .
$$

We infer that $\int_{\Omega \times T} \rho_{\varepsilon}(x)\left|\frac{\partial u_{\varepsilon}}{\partial t}\right|^{2} \mathrm{~d} x \mathrm{~d} t$ is bounded (because by (2.7), $\rho_{\varepsilon}$ is bounded from below by a positive constant) and then, coming back to (3.25), that

$$
\int_{\Omega} \rho_{\varepsilon}(x)\left|\frac{\partial u_{\varepsilon}}{\partial t}(x, \tau)\right|^{2} \mathrm{~d} x+\int_{\Omega} a_{\varepsilon}\left|\nabla u_{\varepsilon}(x, \tau)\right|^{2} \mathrm{~d} x \leq C, \quad \forall \tau \in[0, T],
$$

yielding (3.14), the boundedness of $\left(u_{\varepsilon}\right)$ in $L^{\infty}\left(0, T ; H_{0}^{1}(\Omega)\right)$, and up to a subsequence the convergence (3.15).

The assertion (3.16) follows from (3.3), the first line of (3.5) and (3.14).

Let us assume that $\gamma>0$. Then, by (2.9), (3.14), (3.15), (3.16) and the third line of (3.5), the sequence $\left(\tilde{v}_{\varepsilon}\right)$ is bounded in $L^{2}(\Omega \times T)$ (resp. in $L^{\infty}\left(0, T ; L^{2}(\Omega)\right)$ if $\left.n=2\right)$ thus converges weakly (resp. star-weakly), up to a subsequence, to some $v \in L^{2}(\Omega \times T)$ (resp. $L^{\infty}\left(0, T ; L^{2}(\Omega)\right)$ if $\left.n=2\right)$, that is (3.19). We infer from the fourth line of $(3.5)$

$$
\int_{\Omega \times T}\left|\tilde{v}_{\varepsilon}\right|^{2} \mathrm{~d} m_{\varepsilon} \mathrm{d} t \leq C, \quad\left(\text { resp. } \quad \int\left|\tilde{v}_{\varepsilon}(x, \tau)\right|^{2} \mathrm{~d} m_{\varepsilon} \leq C, \forall \tau \in[0, T]\right)
$$

hence we can apply Lemma 3.2 with $\nu_{\varepsilon}:=m_{\varepsilon} \otimes \mathcal{L}^{1} L_{(0, T)}$ (which by (3.2) converges star-weakly to the Lebesgue measure on $\Omega \times T)$ and $f_{\varepsilon}:=\tilde{v}_{\varepsilon}$ : we deduce that the sequence of measures $\left(\tilde{v}_{\varepsilon} m_{\varepsilon} \otimes \mathcal{L}^{1} L_{(0, T)}\right)$ is bounded in $\mathcal{M}(\overline{\Omega \times T})$ (resp. in $L^{\infty}(0, T ; \mathcal{M}(\bar{\Omega}))$ ) and weak-star converges, up to a subsequence, to a measure of the type $w \mathrm{~d} x \mathrm{~d} t$ with $w \in L^{2}(\Omega \times T)$. By passing to the limit as $\varepsilon \rightarrow 0$ in the following estimate

$$
\left|\int_{\Omega \times T} \varphi \tilde{v}_{\varepsilon} \mathrm{d} m_{\varepsilon} \mathrm{d} t-\int_{\Omega \times T} \varphi \tilde{v}_{\varepsilon} \mathrm{d} x \mathrm{~d} t\right| \leq C \varepsilon
$$

holding in view of the definitions (3.1) and (3.4), for any $\varphi \in C(\overline{\Omega \times T})$ we deduce $w=v$, hence

$$
\begin{aligned}
\tilde{v}_{\varepsilon} \mathrm{d} m_{\varepsilon} \mathrm{d} t \stackrel{\star}{*} v \mathrm{~d} x \mathrm{~d} t, & \text { star-weakly in } \mathcal{M}(\overline{\Omega \times T}), \\
& \left(\text { resp. star-weakly in } L^{\infty}(0, T, \mathcal{M}(\bar{\Omega})) \text { if } n=2\right) .
\end{aligned}
$$

By (3.1), (3.14) and the second line of (3.5) we have

$$
\begin{aligned}
\int_{\Omega \times T}\left|u_{\varepsilon}-\tilde{v}_{\varepsilon}\right|^{2} \mathrm{~d} m_{\varepsilon} \mathrm{d} t & =\frac{3}{4 \pi} \frac{\varepsilon^{3}}{r_{\varepsilon}^{3}} \int_{B_{\varepsilon} \times(0, T)}\left|u_{\varepsilon}-\tilde{v}_{\varepsilon}\right|^{2} \mathrm{~d} x \mathrm{~d} t \leq C \frac{\varepsilon^{3}}{r_{\varepsilon}^{3}} r_{\varepsilon}^{2} \int_{B_{\varepsilon} \times(0, T)}\left|\nabla u_{\varepsilon}\right|^{2} \mathrm{~d} x \mathrm{~d} t \\
& =C \frac{\varepsilon^{3}}{r_{\varepsilon}} \frac{1}{a_{1 \varepsilon}} \int_{B_{\varepsilon} \times(0, T)} a_{1 \varepsilon}\left|\nabla u_{\varepsilon}\right|^{2} \mathrm{~d} x \mathrm{~d} t \leq C \frac{\varepsilon^{3}}{r_{\varepsilon}} \frac{1}{a_{1 \varepsilon}}, \\
& \left(\text { resp. } \int_{\Omega}\left|u_{\varepsilon}(x, \tau)-\tilde{v}_{\varepsilon}(x, \tau)\right|^{2} \mathrm{~d} m_{\varepsilon}(x) \leq C \frac{\varepsilon^{3}}{r_{\varepsilon}} \frac{1}{a_{1 \varepsilon}}, \forall \tau \in[0, T] \text { if } n=2\right) .
\end{aligned}
$$


The estimate (3.17) follows from (3.27), (3.30) and the inequality

$$
\begin{gathered}
\int_{\Omega \times T}\left|u_{\varepsilon}\right|^{2} \mathrm{~d} m_{\varepsilon} \mathrm{d} t \leq 2 \int_{\Omega \times T}\left|u_{\varepsilon}-\tilde{v}_{\varepsilon}\right|^{2} \mathrm{~d} m_{\varepsilon} \mathrm{d} t+2 \int_{\Omega \times T}\left|\tilde{v}_{\varepsilon}\right|^{2} \mathrm{~d} m_{\varepsilon} \mathrm{d} t \\
\left(\begin{array}{c}
\operatorname{resp} . \int_{\Omega}\left|u_{\varepsilon}(x, \tau)\right|^{2} \mathrm{~d} m_{\varepsilon} \leq 2 \int_{\Omega}\left|u_{\varepsilon}(x, \tau)-\tilde{v}_{\varepsilon}(x, \tau)\right|^{2} \mathrm{~d} m_{\varepsilon}(x)+2 \int_{\Omega}\left|\tilde{v}_{\varepsilon}(x, \tau)\right|^{2} \mathrm{~d} m_{\varepsilon}(x), \\
\forall \tau \in[0, T] \text { if } n=2
\end{array}\right) .
\end{gathered}
$$

By (3.17) we can apply Lemma 3.2 with $\nu_{\varepsilon}:=m_{\varepsilon} \otimes \mathcal{L}^{1}\left\lfloor_{(0, T)}\right.$ and $f_{\varepsilon}:=u_{\varepsilon}$. We infer, up to a subsequence,

$$
\begin{aligned}
u_{\varepsilon} \mathrm{d} m_{\varepsilon} \mathrm{d} t \stackrel{\star}{\rightarrow} g \mathrm{~d} x \mathrm{~d} t, & \text { star-weakly in } \mathcal{M}(\overline{\Omega \times T}), \\
& \left(\text { resp. star-weakly in } L^{\infty}(0, T, \mathcal{M}(\bar{\Omega})) \text { if } n=2\right),
\end{aligned}
$$

for some $g \in L^{2}(\Omega \times T)$. Finally, using the last line of Lemma 3.2 with $\nu_{\varepsilon}:=m_{\varepsilon} \otimes \mathcal{L}^{1}\left\lfloor_{(0, T)}\right.$ and $f_{\varepsilon}:=u_{\varepsilon}-\tilde{v}_{\varepsilon}$, taking into account $(2.7),(3.29),(3.30),(3.32)$ and the assumption $\gamma>0$, we obtain

$$
\int_{\Omega \times T}|g-v|^{2} \mathrm{~d} x \mathrm{~d} t \leq \liminf _{\varepsilon \rightarrow 0} \int_{\Omega \times T}\left|u_{\varepsilon}-\tilde{v}_{\varepsilon}\right|^{2} \mathrm{~d} m_{\varepsilon} \mathrm{d} t \leq C \liminf _{\varepsilon \rightarrow 0} \frac{\varepsilon^{3}}{r_{\varepsilon}} \frac{1}{a_{1 \varepsilon}}=0,
$$

hence $g=v$ and (3.18) follows from (3.32).

\section{Proof of Theorem 2.1}

As in [1], the key point of the proof consists of the construction of an appropriate sequence of oscillating test functions $\left(\Phi_{\varepsilon}\right)$ by which we will multiply (2.3), then pass to the limit as $\varepsilon \rightarrow 0$ in accordance with the convergences stated in Proposition 3.3 to get a weak formulation of the limit problem. We assume first that $\gamma>0$ (the case $\gamma=0$, much easier, is commented at the end of the section). Fixing two regular functions $\varphi, \psi \in C^{\infty}(\overline{\Omega \times(0, T)})$ such that $\varphi=\psi=0$ on $\partial \Omega \times[0, T] \cup \bar{\Omega} \times\{T\}$ and (if $\left.n=2\right) \frac{\partial \varphi}{\partial t}=\frac{\partial \psi}{\partial t}=0$ on $\bar{\Omega} \times\{T\}$, we set

$$
\begin{aligned}
d_{\varepsilon}(x) & :=\operatorname{dist}\left(x,\left\{\varepsilon i, i \in Z^{3}\right\}\right), \\
C_{\varepsilon} & :=\bigcup_{i \in I_{\varepsilon}} B_{R_{\varepsilon}}^{i} \backslash B_{r_{\varepsilon}}^{i}=\left\{x \in \Omega, \quad r_{\varepsilon}<d_{\varepsilon}(x)<R_{\varepsilon}\right\},
\end{aligned}
$$

and define the test functions $\left(\Phi_{\varepsilon}\right)$ by

$$
\Phi_{\varepsilon}(x, t):=\left(1-\theta_{\varepsilon}(x)\right) \varphi(x, t)+\theta_{\varepsilon}(x)\left(\varphi_{\varepsilon}+\psi_{\varepsilon}\right)(x, t),
$$

where

$$
\begin{aligned}
& \varphi_{\varepsilon}(x, t):=\sum_{i \in I_{\varepsilon}}\left(f_{B_{r_{\varepsilon}}^{i}} \varphi(s, t) \mathrm{d} s\right) 1_{Y_{\varepsilon}^{i}}(x), \\
& \psi_{\varepsilon}(x, t):=\sum_{i \in I_{\varepsilon}}\left(f_{B_{r_{\varepsilon}}^{i}} \psi(s, t) \mathrm{d} s\right) 1_{Y_{\varepsilon}^{i}}(x),
\end{aligned}
$$


and $\theta_{\varepsilon}: \Omega \rightarrow[0,1]$ is given by

$$
\begin{array}{lll}
\theta_{\varepsilon}(x):=1 \quad \text { if } d_{\varepsilon}(x)<r_{\varepsilon} ; & \theta_{\varepsilon}(x):=0 & \text { if } d_{\varepsilon}(x)>R_{\varepsilon} ; \\
\theta_{\varepsilon}(x):=\frac{r_{\varepsilon}}{d_{\varepsilon}(x)} \frac{R_{\varepsilon}-d_{\varepsilon}(x)}{R_{\varepsilon}-r_{\varepsilon}} & \text { otherwise. }
\end{array}
$$

Notice that $\theta_{\varepsilon}$ is the solution on each subset $B_{R_{\varepsilon}}^{i} \backslash B_{r_{\varepsilon}}^{i}$ of the minimization problem

$$
\min \left\{\int_{B_{R_{\varepsilon}}^{i} \backslash B_{r_{\varepsilon}}^{i}}|\nabla \theta(x)|^{2} \mathrm{~d} x, \theta \in H^{1}\left(B_{R_{\varepsilon}}^{i} \backslash B_{r_{\varepsilon}}^{i}\right), \theta=1 \text { on } \partial B_{r_{\varepsilon}}^{i}, \theta=0 \text { on } \partial B_{R_{\varepsilon}}^{i}\right\} .
$$

By multiplying equation (2.3) by $\Phi_{\varepsilon}$ and integrating it by parts over $\Omega \times(0, T)$, we get if $n=1$ :

$$
-\int_{\Omega} \rho_{\varepsilon}(x) U_{0}(x) \Phi_{\varepsilon}(x, 0) \mathrm{d} x-\int_{0}^{T} \int_{\Omega} \rho_{\varepsilon}(x) u_{\varepsilon} \frac{\partial \Phi_{\varepsilon}}{\partial t} \mathrm{~d} x \mathrm{~d} t+\int_{0}^{T} \int_{\Omega} a_{\varepsilon} \nabla u_{\varepsilon} \nabla \Phi_{\varepsilon} \mathrm{d} x \mathrm{~d} t=\int_{0}^{T} \int_{\Omega} f \Phi_{\varepsilon} \mathrm{d} x \mathrm{~d} t,
$$

and if $n=2$ :

$$
\begin{aligned}
& -\int_{\Omega} \rho_{\varepsilon}(x) V_{0}(x) \Phi_{\varepsilon}(x, 0) \mathrm{d} x+\int_{\Omega} \rho_{\varepsilon}(x) U_{0}(x) \frac{\partial \Phi_{\varepsilon}}{\partial t}(x, 0) \mathrm{d} x \\
& \quad+\int_{0}^{T} \int_{\Omega} \rho_{\varepsilon}(x) u_{\varepsilon} \frac{\partial^{2} \Phi_{\varepsilon}}{\partial t^{2}} \mathrm{~d} x \mathrm{~d} t+\int_{0}^{T} \int_{\Omega} a_{\varepsilon} \nabla u_{\varepsilon} \nabla \Phi_{\varepsilon} \mathrm{d} x \mathrm{~d} t=\int_{0}^{T} \int_{\Omega} f \Phi_{\varepsilon} \mathrm{d} x \mathrm{~d} t .
\end{aligned}
$$

By (3.3), (4.1) and (4.2), for all $\tau \in[0, T]$ and all $m \in\{0,1,2\}$ there holds

$$
\begin{aligned}
\int_{\Omega}\left|\frac{\partial^{m}}{\partial t^{m}} \Phi_{\varepsilon}(x, \tau)-\frac{\partial^{m}}{\partial t^{m}} \varphi(x, \tau)\right|^{2} \mathrm{~d} x & \leq \int_{\left(C_{\varepsilon} \cup B_{\varepsilon}\right)}\left|\frac{\partial^{m}}{\partial t^{m}} \Phi_{\varepsilon}(x, \tau)-\frac{\partial^{m}}{\partial t^{m}} \varphi(x, \tau)\right|^{2} \mathrm{~d} x \\
& \leq C\left|C_{\varepsilon} \cup B_{\varepsilon}\right| \leq C \frac{R_{\varepsilon}^{3}}{\varepsilon^{3}} \rightarrow 0
\end{aligned}
$$

hence

$$
\begin{aligned}
& \Phi_{\varepsilon} \rightarrow \varphi \text { strongly in } L^{2}(\Omega \times T) ; \quad \Phi_{\varepsilon}(., 0) \rightarrow \varphi(., 0) \text { strongly in } L^{2}(\Omega) ; \\
& \frac{\partial^{n} \Phi_{\varepsilon}}{\partial t^{n}} \rightarrow \frac{\partial^{n} \varphi}{\partial t^{n}} \text { strongly in } L^{2}(\Omega \times T) ; \frac{\partial^{n} \Phi_{\varepsilon}}{\partial t^{n}}(x, 0) \rightarrow \frac{\partial^{n} \varphi}{\partial t^{n}}(x, 0) \text { strongly in } L^{2}(\Omega) .
\end{aligned}
$$

To determine the limit behaviour of the sequences $\left(\rho_{\varepsilon}(x) \Phi_{\varepsilon}(x, 0)\right)$ and $\left(\rho_{\varepsilon}(x) \frac{\partial \Phi_{\varepsilon}}{\partial t}(x, 0)\right)$, we fix $g \in C(\bar{\Omega})$ and write (see (3.1), (4.2))

$$
\begin{aligned}
\int_{\Omega} \rho_{\varepsilon}(x) \Phi_{\varepsilon}(x, 0) g(x) \mathrm{d} x= & \int_{\Omega} \rho_{0} \Phi_{\varepsilon}(x, 0) g(x) 1_{\Omega \backslash B_{\varepsilon}}(x) \mathrm{d} x \\
& +\rho_{1 \varepsilon} \frac{4 \pi r_{\varepsilon}^{3}}{3 \varepsilon^{3}} \int\left(\varphi_{\varepsilon}(x, 0)+\psi_{\varepsilon}(x, 0)\right) g(x) \mathrm{d} m_{\varepsilon}, \\
\int_{\Omega} \rho_{\varepsilon}(x) \frac{\partial \Phi_{\varepsilon}}{\partial t}(x, 0) g(x) \mathrm{d} x= & \int_{\Omega} \rho_{0} \frac{\partial \Phi_{\varepsilon}}{\partial t}(x, 0) g(x) 1_{\Omega \backslash B_{\varepsilon}}(x) \mathrm{d} x \\
& +\rho_{1 \varepsilon} \frac{4 \pi r_{\varepsilon}^{3}}{3 \varepsilon^{3}} \int\left(\frac{\partial \varphi_{\varepsilon}}{\partial t}(x, 0)+\frac{\partial \psi_{\varepsilon}}{\partial t}(x, 0)\right) g(x) \mathrm{d} m_{\varepsilon} .
\end{aligned}
$$


By (4.9) and the strong convergences in $L^{2}(\Omega)$ of the sequence $\left(g(x) 1_{\Omega \backslash B_{\varepsilon}}(x)\right)$ to $g$ (due to $\left|B_{\varepsilon}\right| \rightarrow 0$ ), there holds

$$
\begin{aligned}
& \lim _{\varepsilon \rightarrow 0} \int_{\Omega} \rho_{0} \Phi_{\varepsilon}(x, 0) g(x) 1_{\Omega \backslash B_{\varepsilon}}(x) \mathrm{d} x=\int_{\Omega} \rho_{0} \varphi(x, 0) g(x) \mathrm{d} x, \\
& \lim _{\varepsilon \rightarrow 0} \int_{\Omega} \rho_{0} \frac{\partial \Phi_{\varepsilon}}{\partial t}(x, 0) g(x) 1_{\Omega \backslash B_{\varepsilon}}(x) \mathrm{d} x=\int_{\Omega} \rho_{0} \frac{\partial \varphi}{\partial t}(x, 0) g(x) \mathrm{d} x .
\end{aligned}
$$

From (2.7), (3.2) and the following estimates, holding on $B_{\varepsilon}$ in view of (4.2), (4.3)

$$
\begin{aligned}
& \left|\varphi_{\varepsilon}(x, 0)+\psi_{\varepsilon}(x, 0)-(\varphi(x, 0)+\psi(x, 0))\right| \leq C r_{\varepsilon}, \forall x \in B_{\varepsilon}, \\
& \left|\frac{\partial^{n} \Phi_{\varepsilon}}{\partial t^{n}}-\frac{\partial^{n}(\varphi+\psi)}{\partial t^{n}}\right|(x, t)=\left|\frac{\partial^{n}\left(\varphi_{\varepsilon}+\psi_{\varepsilon}\right)}{\partial t^{n}}-\frac{\partial^{n}(\varphi+\psi)}{\partial t^{n}}\right|(x, t) \leq C r_{\varepsilon}, \quad \forall x \in B_{\varepsilon},
\end{aligned}
$$

we infer

$$
\begin{aligned}
& \lim _{\varepsilon \rightarrow 0} \rho_{1 \varepsilon} \frac{4 \pi}{3} \frac{r_{\varepsilon}^{3}}{\varepsilon^{3}} \int\left(\varphi_{\varepsilon}(x, 0)+\psi_{\varepsilon}(x, 0)\right) g(x) \mathrm{d} m_{\varepsilon}=\bar{\rho}_{1} \int_{\Omega}(\varphi(x, 0)+\psi(x, 0)) g(x) \mathrm{d} x, \\
& \lim _{\varepsilon \rightarrow 0} \rho_{1 \varepsilon} \frac{4 \pi}{3} \frac{r_{\varepsilon}^{3}}{\varepsilon^{3}} \int\left(\frac{\partial \varphi_{\varepsilon}}{\partial t}(x, 0)+\frac{\partial \psi_{\varepsilon}}{\partial t}(x, 0)\right) g(x) \mathrm{d} m_{\varepsilon}=\bar{\rho}_{1} \int_{\Omega}\left(\frac{\partial \varphi}{\partial t}(x, 0)+\frac{\partial \psi}{\partial t}(x, 0)\right) g(x) \mathrm{d} x,
\end{aligned}
$$

and deduce from (4.10), (4.11), (4.13) and the arbitrary nature of $g$ that

$$
\begin{aligned}
& \rho_{\varepsilon}(x) \Phi_{\varepsilon}(x, 0) \stackrel{\star}{\rightarrow} \rho_{0} \varphi(x, 0)+\bar{\rho}_{1}(\varphi(x, 0)+\psi(x, 0)) \quad \text { star-weakly in } \mathcal{M}(\bar{\Omega}), \\
& \rho_{\varepsilon}(x) \frac{\partial \Phi_{\varepsilon}}{\partial t}(x, 0) \stackrel{\star}{\rightarrow} \rho_{0} \frac{\partial \varphi}{\partial t}(x, 0)+\bar{\rho}_{1}\left(\frac{\partial \varphi}{\partial t}(x, 0)+\frac{\partial \psi}{\partial t}(x, 0)\right) \quad \text { star-weakly in } \mathcal{M}(\bar{\Omega}) .
\end{aligned}
$$

It follows from (4.9) and (4.14) that (see (2.4))

$$
\begin{aligned}
& \lim _{\varepsilon \rightarrow 0} \int_{\Omega} \rho_{\varepsilon}(x) U_{0}(x) \Phi_{\varepsilon}(x, 0) \mathrm{d} x=\int_{\Omega} U_{0}(x)\left(\rho_{0} \varphi(x, 0)+\bar{\rho}_{1}(\varphi(x, 0)+\psi(x, 0))\right) \mathrm{d} x, \quad(n=1), \\
& \lim _{\varepsilon \rightarrow 0} \int_{\Omega} \rho_{\varepsilon}(x) U_{0}(x) \frac{\partial \Phi_{\varepsilon}}{\partial t}(x, 0) \mathrm{d} x=\int_{\Omega} U_{0}(x)\left(\rho_{0} \frac{\partial \varphi}{\partial t}(x, 0)+\bar{\rho}_{1}\left(\frac{\partial \varphi}{\partial t}(x, 0)+\frac{\partial \psi}{\partial t}(x, 0)\right)\right) \mathrm{d} x,(n=2), \\
& \lim _{\varepsilon \rightarrow 0} \int_{\Omega} \rho_{\varepsilon}(x) V_{0}(x) \Phi_{\varepsilon}(x, 0) \mathrm{d} x=\int_{\Omega} V_{0}(x)\left(\rho_{0} \varphi(x, 0)+\bar{\rho}_{1}(\varphi(x, 0)+\psi(x, 0))\right) \mathrm{d} x, \quad(n=2), \\
& \lim _{\varepsilon \rightarrow 0} \int_{0}^{T} \int_{\Omega} f \Phi_{\varepsilon} \mathrm{d} x \mathrm{~d} t=\int_{0}^{T} \int_{\Omega} f \varphi \mathrm{d} x \mathrm{~d} t .
\end{aligned}
$$

We infer from (4.9), (4.12) and the following convergences (due to (2.7), (2.8), (3.15) and (3.18))

$$
\begin{aligned}
& \rho_{\varepsilon}(x) u_{\varepsilon}(x, t) 1_{B_{\varepsilon}}(x) \stackrel{\star}{\star} \bar{\rho}_{1} v \quad \text { star-weakly in } \mathcal{M}(\overline{\Omega \times T}), \\
& \rho_{\varepsilon}(x) u_{\varepsilon}(x, t) 1_{\Omega \backslash B_{\varepsilon}}(x) \rightarrow \rho_{0} u \quad \text { weakly in } L^{2}(\Omega \times T),
\end{aligned}
$$

that

$$
\begin{aligned}
\lim _{\varepsilon \rightarrow 0} \int_{0}^{T} \int_{\Omega} \rho_{\varepsilon}(x) u_{\varepsilon} \frac{\partial^{n} \Phi_{\varepsilon}}{\partial t^{n}} \mathrm{~d} x \mathrm{~d} t & =\lim _{\varepsilon \rightarrow 0} \int_{0}^{T} \int_{\Omega} \rho_{\varepsilon}(x) u_{\varepsilon} 1_{\Omega \backslash B_{\varepsilon}}(x) \frac{\partial^{n} \Phi_{\varepsilon}}{\partial t^{n}} \mathrm{~d} x \mathrm{~d} t+\int_{0}^{T} \int_{\Omega} \rho_{\varepsilon}(x) u_{\varepsilon} 1_{B_{\varepsilon}}(x) \frac{\partial^{n} \Phi_{\varepsilon}}{\partial t^{n}} \mathrm{~d} x \mathrm{~d} t \\
& =\int_{0}^{T} \int_{\Omega}\left(\rho_{0} u \frac{\partial^{n} \varphi}{\partial t^{n}}+\bar{\rho}_{1} v \frac{\partial^{n}(\varphi+\psi)}{\partial t^{n}}\right) \mathrm{d} x \mathrm{~d} t .
\end{aligned}
$$


Noticing that $\nabla \Phi_{\varepsilon}=0$ on $B_{\varepsilon}$, we split the remaining term of (4.6) (or (4.7)) into a sum of two terms:

$$
\begin{aligned}
& \int_{0}^{T} \int_{\Omega} \nabla u_{\varepsilon} \nabla \Phi_{\varepsilon} \mathrm{d} x \mathrm{~d} t=I_{1 \varepsilon}+I_{2 \varepsilon} \\
& I_{1 \varepsilon}:=\int_{0}^{T} \int_{\Omega \backslash\left(C_{\varepsilon} \cup B_{\varepsilon}\right)} \nabla u_{\varepsilon} \nabla \varphi \mathrm{d} x \mathrm{~d} t, \quad I_{2 \varepsilon}:=\int_{0}^{T} \int_{C_{\varepsilon}} \nabla u_{\varepsilon} \nabla \Phi_{\varepsilon} \mathrm{d} x \mathrm{~d} t .
\end{aligned}
$$

By (3.15) and by the strong convergence in $L^{2}$ of $\nabla \varphi \mathbf{1}_{\Omega \backslash\left(C_{\varepsilon} \cup B_{\varepsilon}\right)}$ to $\nabla \varphi$ (due to (3.3)), we obtain

$$
\lim _{\varepsilon \rightarrow 0} I_{1 \varepsilon}=\int_{0}^{T} \int_{\Omega} \nabla u \nabla \varphi \mathrm{d} x \mathrm{~d} t
$$

With regard to $I_{2 \varepsilon}$, we shall prove (see below) that

$$
\lim _{\varepsilon \rightarrow 0}\left(I_{2 \varepsilon}-\int_{0}^{T} \int_{C_{\varepsilon}} \psi_{\varepsilon} \nabla u_{\varepsilon} \nabla \theta_{\varepsilon} \mathrm{d} x \mathrm{~d} t\right)=0
$$

then, noticing that on each subset $B_{R_{\varepsilon}}^{i} \backslash B_{r_{\varepsilon}}^{i}$ the function $\psi_{\varepsilon}(x)$ is constant and the function $\theta_{\varepsilon}$ and the field $\nabla \theta_{\varepsilon}$ are given in terms of the local spherical coordinates $\left(x_{1}-i_{1}=r \sin \theta_{1} \cos \theta_{2}, x_{2}-i_{2}=r \sin \theta_{1} \sin \theta_{2}, x_{3}-i_{3}=\right.$ $\left.r \cos \theta_{1}\right)$ by

$$
\theta_{\varepsilon}\left(r, \theta_{1}, \theta_{2}\right)=\frac{r_{\varepsilon}}{r} \frac{R_{\varepsilon}-r}{R_{\varepsilon}-r_{\varepsilon}} ; \quad \nabla \theta_{\varepsilon}\left(r, \theta_{1}, \theta_{2}\right)=-\frac{1}{r^{2}} \frac{r_{\varepsilon} R_{\varepsilon}}{R_{\varepsilon}-r_{\varepsilon}} n\left(\theta_{1}, \theta_{2}\right)
$$

where $n\left(\theta_{1}, \theta_{2}\right)$ is the unit vector in the direction given by the angles $\theta_{1}, \theta_{2}$, we infer (see (3.4))

$$
\begin{aligned}
\int_{0}^{T} \int_{B_{R_{\varepsilon}}^{i} \backslash B_{r_{\varepsilon}}^{i}} \psi_{\varepsilon} \nabla u_{\varepsilon} \nabla \theta_{\varepsilon} \mathrm{d} x & =\int_{0}^{T} \int_{B_{R_{\varepsilon}}^{i} \backslash B_{r_{\varepsilon}}^{i}} \psi_{\varepsilon} \nabla u_{\varepsilon} \nabla \theta_{\varepsilon} r^{2} \sin \theta_{1} \mathrm{~d} r \mathrm{~d} \theta_{1} \mathrm{~d} \theta_{2} \mathrm{~d} t \\
& =\frac{r_{\varepsilon} R_{\varepsilon}}{R_{\varepsilon}-r_{\varepsilon}} \int_{0}^{T} \int_{\theta_{1}=0}^{\pi} \int_{\theta_{2}=0}^{2 \pi} \int_{r=r_{\varepsilon}}^{R_{\varepsilon}}\left(-\frac{\partial u_{\varepsilon}}{\partial r}\left(r, \theta_{1}, \theta_{2}, t\right)\right) \psi_{\varepsilon} \mathrm{d} r \sin \theta_{1} \mathrm{~d} \theta_{1} \mathrm{~d} \theta_{2} \mathrm{~d} t \\
& =\frac{r_{\varepsilon} R_{\varepsilon}}{R_{\varepsilon}-r_{\varepsilon}} \int_{0}^{T} \int_{0}^{\pi} \int_{0}^{2 \pi}\left(u_{\varepsilon}\left(r_{\varepsilon}, \theta_{1}, \theta_{2}, t\right)-u_{\varepsilon}\left(R_{\varepsilon}, \theta_{1}, \theta_{2}, t\right)\right) \psi_{\varepsilon} \sin \theta_{1} \mathrm{~d} \theta_{1} \mathrm{~d} \theta_{2} \mathrm{~d} t \\
& =4 \pi \frac{r_{\varepsilon} R_{\varepsilon}}{R_{\varepsilon}-r_{\varepsilon}} \int_{0}^{T}\left(\tilde{v}_{\varepsilon}-\tilde{u}_{\varepsilon}\right) \psi_{\varepsilon} \mathrm{d} t \\
& =\frac{4 \pi}{\varepsilon^{3}} \frac{r_{\varepsilon} R_{\varepsilon}}{R_{\varepsilon}-r_{\varepsilon}} \int_{0}^{T} \int_{Y_{\varepsilon}^{i}}\left(\tilde{v}_{\varepsilon}-\tilde{u}_{\varepsilon}\right) \psi_{\varepsilon} \mathrm{d} x \mathrm{~d} t
\end{aligned}
$$

then, adding up (4.20) over $i \in I_{\varepsilon}$,

$$
\int_{0}^{T} \int_{C_{\varepsilon}} \psi_{\varepsilon} \nabla u_{\varepsilon} \nabla \theta_{\varepsilon} \mathrm{d} x \mathrm{~d} t=\frac{4 \pi}{\varepsilon^{3}} \frac{r_{\varepsilon} R_{\varepsilon}}{R_{\varepsilon}-r_{\varepsilon}} \int_{0}^{T} \int_{\Omega}\left(\tilde{v}_{\varepsilon}-\tilde{u}_{\varepsilon}\right) \psi_{\varepsilon} \mathrm{d} x \mathrm{~d} t .
$$

By (3.15), (3.16), (3.19) and the estimate $\left|\psi-\psi_{\varepsilon}\right| \leq C \varepsilon$, there holds

$$
\lim _{\varepsilon \rightarrow 0} \int_{0}^{T} \int_{\Omega}\left(\tilde{v}_{\varepsilon}-\tilde{u}_{\varepsilon}\right) \psi_{\varepsilon} \mathrm{d} x \mathrm{~d} t=\int_{0}^{T} \int_{\Omega}(v-u) \psi \mathrm{d} x \mathrm{~d} t .
$$

Assuming first that $0<\gamma<+\infty$, we deduce from (2.9), (4.19), (4.21) and (4.22)

$$
\lim _{\varepsilon \rightarrow 0} I_{2 \varepsilon}=4 \pi \gamma \int_{0}^{T} \int_{\Omega}(v-u) \psi \mathrm{d} x \mathrm{~d} t
$$


and passing to the limit as $\varepsilon \rightarrow 0$ in (4.6) and in (4.7), taking into account (4.15), (4.16), (4.17), (4.18) and (4.23), we obtain if $n=1$

$$
\begin{array}{rl}
-\int_{\Omega} U_{0}(x)\left(\rho_{0} \varphi+\bar{\rho}_{1}(\varphi+\psi)\right)(x, 0) \mathrm{d} & x-\int_{0}^{T} \int_{\Omega}\left(\rho_{0} u \frac{\partial \varphi}{\partial t}+\bar{\rho}_{1} v \frac{\partial(\varphi+\psi)}{\partial t}\right) \mathrm{d} x \mathrm{~d} t \\
& +\int_{0}^{T} \int_{\Omega} \nabla u \nabla \varphi \mathrm{d} x \mathrm{~d} t+4 \pi \gamma \int_{0}^{T} \int_{\Omega}(v-u) \psi \mathrm{d} x \mathrm{~d} t=\int_{0}^{T} \int_{\Omega} f \varphi \mathrm{d} x \mathrm{~d} t,
\end{array}
$$

and if $n=2$,

$$
\begin{aligned}
-\int_{\Omega} V_{0}(x)\left(\rho_{0} \varphi+\bar{\rho}_{1}(\varphi+\psi)\right)(x, 0) \mathrm{d} x+\int_{\Omega} U_{0}(x)\left(\rho_{0} \frac{\partial \varphi}{\partial t}+\bar{\rho}_{1} \frac{\partial(\varphi+\psi)}{\partial t}\right)(x, 0) \mathrm{d} x & \\
& +\int_{0}^{T} \int_{\Omega}\left(\rho_{0} u \frac{\partial^{2} \varphi}{\partial t^{2}}+\bar{\rho}_{1} v \frac{\partial^{2}(\varphi+\psi)}{\partial t^{2}}\right) \mathrm{d} x \mathrm{~d} t+\int_{0}^{T} \int_{\Omega} \nabla u \nabla \varphi \mathrm{d} x \mathrm{~d} t \\
& \quad+4 \pi \gamma \int_{0}^{T} \int_{\Omega}(v-u) \psi \mathrm{d} x \mathrm{~d} t=\int_{0}^{T} \int_{\Omega} f \varphi \mathrm{d} x \mathrm{~d} t .
\end{aligned}
$$

By choosing successively $\psi=-\varphi$ and $\varphi=0$ in (4.24), (4.25) we deduce from the arbitrary nature of $\varphi, \psi$ and from the standard regularity results (see [8]) that the couple $(u, v)$ is a solution of:

$$
\begin{cases}\rho_{0} \frac{\partial^{n} u}{\partial t^{n}}-\Delta u+4 \pi \gamma(u-v)=f & \text { on } \Omega \times T, \\ \bar{\rho}_{1} \frac{\partial^{n} v}{\partial t^{n}}+4 \pi \gamma(v-u)=0 & \text { on } \Omega \times T, \\ (u, v) \in \mathcal{D}_{n}^{\mathrm{eff}}, & \end{cases}
$$

where $\mathcal{D}_{n}^{\text {eff }}$ is defined by (2.11). The proof of Theorem 2.1 in the case $0<\gamma<+\infty$ is achieved.

Assuming now $\gamma=+\infty$, we first notice that by (4.6), (4.7), (4.15), (4.16), (4.17) and (4.18) the sequence $I_{2 \varepsilon}$ admits a finite limit as $\varepsilon \rightarrow 0$. Since $\gamma$ is infinite, we deduce from (4.19), (4.21) and (4.22) that $\int_{0}^{T} \int_{\Omega}(v-$ $u) \psi \mathrm{d} x \mathrm{~d} t=0$, hence $u=v$. Next we substitute 0 for $\psi$ in (4.3) and pass to the limit as $\varepsilon \rightarrow 0$ in (4.6) and (4.7). By (4.15), (4.16), (4.18), (4.19), we obtain for $n=1$

$$
-\int_{\Omega} U_{0}(x)\left(\rho_{0}+\bar{\rho}_{1}\right) \varphi(x, 0) \mathrm{d} x-\int_{0}^{T} \int_{\Omega}\left(\rho_{0}+\bar{\rho}_{1}\right) u \frac{\partial \varphi}{\partial t} \mathrm{~d} x \mathrm{~d} t+\int_{0}^{T} \int_{\Omega} \nabla u \nabla \varphi \mathrm{d} x \mathrm{~d} t=\int_{0}^{T} \int_{\Omega} f \varphi \mathrm{d} x \mathrm{~d} t,
$$

and for $n=2$,

$$
\begin{aligned}
-\int_{\Omega} V_{0}(x)\left(\rho_{0}+\bar{\rho}_{1}\right) \varphi(x, 0) \mathrm{d} x+\int_{\Omega} U_{0}(x)\left(\rho_{0}+\bar{\rho}_{1}\right) \frac{\partial \varphi}{\partial t}(x, 0) \mathrm{d} x+\int_{0}^{T} \int_{\Omega}\left(\rho_{0}+\bar{\rho}_{1}\right) u \frac{\partial^{2} \varphi}{\partial t^{2}} \mathrm{~d} x \mathrm{~d} t & \\
& +\int_{0}^{T} \int_{\Omega} \nabla u \nabla \varphi \mathrm{d} x \mathrm{~d} t=\int_{0}^{T} \int_{\Omega} f \varphi \mathrm{d} x \mathrm{~d} t
\end{aligned}
$$

yielding by the same argument the case $\gamma=+\infty$ of Theorem 2.1 .

If $\gamma=0$, the limit equation is obtained by repeating the previous line of reasoning with the test function

$$
\Phi_{\varepsilon}(x, t):=\varphi\left(1-\theta_{\varepsilon}\right) .
$$


Proof of (4.19). By the definitions (4.1)-(4.4), we check easily that

$$
\begin{aligned}
& \int_{0}^{T} \int_{C_{\varepsilon}}\left|\nabla \theta_{\varepsilon}\right|^{2} \mathrm{~d} x \mathrm{~d} t \leq \frac{C}{\varepsilon^{3}} \frac{r_{\varepsilon} R_{\varepsilon}}{R_{\varepsilon}-r_{\varepsilon}} \quad, \quad\left|\varphi-\varphi_{\varepsilon}\right|<C R_{\varepsilon} \text { on } C_{\varepsilon} \times(0, T), \\
& \left|C_{\varepsilon} \times(0, T)\right| \leq C \frac{R_{\varepsilon}^{3}}{\varepsilon^{3}} \quad, \quad \nabla\left(\varphi_{\varepsilon}+\psi_{\varepsilon}-\varphi\right)=-\nabla \varphi \text { on } C_{\varepsilon} \times(0, T), 0 \leq \theta_{\varepsilon} \leq 1 .
\end{aligned}
$$

Since $\nabla \Phi_{\varepsilon}-\nabla \theta_{\varepsilon} \psi_{\varepsilon}=\nabla \varphi+\nabla \theta_{\varepsilon}\left(\varphi_{\varepsilon}-\varphi\right)+\theta_{\varepsilon} \nabla\left(\varphi_{\varepsilon}+\psi_{\varepsilon}-\varphi\right)$, we infer from (2.8), (3.3) and (4.28)

$$
\int_{0}^{T} \int_{C_{\varepsilon}}\left|\nabla \Phi_{\varepsilon}-\nabla \theta_{\varepsilon} \psi_{\varepsilon}\right|^{2} \mathrm{~d} x \mathrm{~d} t \leq C \frac{R_{\varepsilon}^{3}}{\varepsilon^{3}}+\frac{C}{\varepsilon^{3}} \frac{R_{\varepsilon} r_{\varepsilon}}{R_{\varepsilon}-r_{\varepsilon}} R_{\varepsilon}^{2} \longrightarrow_{\varepsilon \rightarrow 0} 0
$$

which proves (4.19), because $\nabla u_{\varepsilon}$ is bounded in $L^{2}(\Omega \times T)$.

\section{Justification of (ii) and (v) of Remark 2.2}

(ii) Assume that $\gamma=0$ and $\bar{\rho}_{1}>0$, then if $n=1$, by (2.7) and (3.20) (resp. by (2.7) and (3.26) if $\left.n=2\right)$ the following estimate holds for all $\tau \in[0, T]$

$$
\begin{aligned}
& \int_{\Omega}\left|u_{\varepsilon}(x, \tau)\right|^{2} \mathrm{~d} m_{\varepsilon} \leq C \int_{\Omega} \rho_{\varepsilon}(x)\left|u_{\varepsilon}(x, \tau)\right|^{2} \mathrm{~d} x \leq C, \\
& \left(\begin{array}{rl}
\text { resp. } \int_{\Omega}\left|u_{\varepsilon}(x, \tau)\right|^{2} \mathrm{~d} m_{\varepsilon} & \leq C \int_{\Omega} \rho_{\varepsilon}(x)\left|u_{\varepsilon}(x, \tau)\right|^{2} \mathrm{~d} x \\
& \left.=C \int_{\Omega} \rho_{\varepsilon}(x)\left|\int_{0}^{\tau} \frac{\partial u_{\varepsilon}}{\partial t}(x, s) \mathrm{d} s+U_{0}(x)\right|^{2} \mathrm{~d} x \leq C, \quad \text { if } n=2\right) .
\end{array}\right.
\end{aligned}
$$

By applying Lemma 3.2 we get (3.18), and we deduce from the fourth line of (3.5) that $\tilde{v}_{\varepsilon}$ is bounded in $L^{2}(\Omega \times T)$, hence converges weakly in $L^{2}(\Omega \times T)$, up to a subsequence, to a function which by virtue of (3.28) is equal to $v$. By choosing test functions defined by (4.2) (instead of (4.27)), and by passing to the limit as $\varepsilon \rightarrow 0$ in (4.6) (resp. in (4.7) if $n=2$ ), we obtain (4.24) (resp. (4.25) if $n=2$ ) and (4.26).

(v) We indicate how to establish the weak convergence in $H_{0}^{1}(\Omega)$, stated in Remark $2.2(\mathrm{v})$, of the sequence $\left(u_{\varepsilon}\right)$ of solutions of $(2.17)$ to the solution $u$ of $(2.18)$. In what follows, when we refer to some formula obtained in the proof of theorem 2.1 , it is to be inferred that symbols of type $\int_{A} \ldots \mathrm{d} m, X, \Omega \ldots$ are substituted for $\int_{A \times(0, T)} \cdots \mathrm{d} m \mathrm{~d} t, L^{p}(0, T ; X), \Omega \times(0, T) \ldots$

We multiply (2.17) by $u_{\varepsilon}$, integrate by parts over $\Omega$ and use Hölder's inequality to find

$$
\int_{\Omega} a_{\varepsilon}\left|\nabla u_{\varepsilon}\right|^{2} \mathrm{~d} x=\int_{\Omega} \rho_{\varepsilon} f . u_{\varepsilon} \leq\left(\int_{\Omega}|f|^{2} \rho_{\varepsilon} \mathrm{d} x\right)^{\frac{1}{2}}\left(\int_{\Omega}\left|u_{\varepsilon}\right|^{2} \rho_{\varepsilon} \mathrm{d} x\right)^{\frac{1}{2}} .
$$

By (2.7) we have $\rho_{\varepsilon}(x) \leq C\left(1+\frac{|\Omega|}{\left|B_{\varepsilon}\right|} 1_{B_{\varepsilon}}\right)$ on $\Omega$, hence by (3.1) and Poincaré's inequality there holds

$$
\int_{\Omega}\left|u_{\varepsilon}\right|^{2} \rho_{\varepsilon} \mathrm{d} x \leq C \int_{\Omega}\left|u_{\varepsilon}\right|^{2} \mathrm{~d} x+C \int\left|u_{\varepsilon}\right|^{2} \mathrm{~d} m_{\varepsilon} \leq C \int_{\Omega}\left|\nabla u_{\varepsilon}\right|^{2} \mathrm{~d} x+C \int\left|u_{\varepsilon}\right|^{2} \mathrm{~d} m_{\varepsilon} .
$$


The estimates (3.5) and Poincaré's inequality yield

$$
\begin{aligned}
\int\left|u_{\varepsilon}\right|^{2} \mathrm{~d} m_{\varepsilon} & \leq 2 \int\left|u_{\varepsilon}-\tilde{v}_{\varepsilon}\right|^{2} \mathrm{~d} m_{\varepsilon}+2 \int\left|\tilde{v}_{\varepsilon}\right|^{2} \mathrm{~d} m_{\varepsilon}=2 \frac{3 \varepsilon^{3}}{4 \pi r_{\varepsilon}^{3}} \int_{B_{\varepsilon}}\left|u_{\varepsilon}-\tilde{v}_{\varepsilon}\right|^{2} \mathrm{~d} x+2 \int_{\Omega}\left|\tilde{v}_{\varepsilon}\right|^{2} \mathrm{~d} x \\
& \leq C \frac{\varepsilon^{3}}{r_{\varepsilon}^{3}} \int_{B_{\varepsilon}}\left|u_{\varepsilon}-\tilde{v}_{\varepsilon}\right|^{2} \mathrm{~d} x+4 \int_{\Omega}\left|\tilde{v}_{\varepsilon}-\tilde{u}_{\varepsilon}\right|^{2} \mathrm{~d} x+4 \int_{\Omega}\left|\tilde{u}_{\varepsilon}-u_{\varepsilon}\right|^{2} \mathrm{~d} x+4 \int_{\Omega}\left|u_{\varepsilon}\right|^{2} \mathrm{~d} x \\
& \leq C\left(\frac{\varepsilon^{3}}{r_{\varepsilon}}+\frac{\varepsilon^{3}}{R_{\varepsilon}}+\varepsilon^{\frac{3}{2}}+1\right) \int_{\Omega}\left|\nabla u_{\varepsilon}\right|^{2} \mathrm{~d} x \\
& \leq C \int_{\Omega}|\nabla u|^{2} \mathrm{~d} x .
\end{aligned}
$$

In the last inequality, we used that $\frac{\varepsilon^{3}}{R_{\varepsilon}} \ll \frac{\varepsilon^{3}}{r_{\varepsilon}} \ll 1$ (because $\gamma>0$, see (2.9)). By the assumption $a_{\varepsilon}>c>0$ (see (2.7)) we have

$$
\int_{\Omega}\left|\nabla\left(u_{\varepsilon}\right)\right|^{2} \mathrm{~d} x \leq C \int_{\Omega} a_{\varepsilon}\left|\nabla\left(u_{\varepsilon}\right)\right|^{2} \mathrm{~d} x .
$$

Collecting (4.29), (4.30), (4.31), (4.32), we infer

$$
\int_{\Omega}\left|u_{\varepsilon}\right|^{2} \rho_{\varepsilon} \mathrm{d} x \leq C\left(\int|f|^{2} \rho_{\varepsilon} \mathrm{d} x\right)^{\frac{1}{2}}\left(\int\left|u_{\varepsilon}\right|^{2} \rho_{\varepsilon} \mathrm{d} x\right)^{\frac{1}{2}}
$$

Since $\bar{\rho}_{1}<+\infty$, the measure $\rho_{\varepsilon} 1_{\Omega} \mathrm{d} x$ is bounded (see (2.7)) hence the quantity $\int|f|^{2} \rho_{\varepsilon} \mathrm{d} x$ is bounded (recall that $f$ is assumed to be continuous). We deduce that $\int_{\Omega}\left|u_{\varepsilon}\right|^{2} \rho_{\varepsilon} \mathrm{d} x$ is bounded and then, by (4.29), (4.31), (4.32) we get

$$
\int_{\Omega}\left|u_{\varepsilon}\right|^{2} \mathrm{~d} x \leq C, \quad \int\left|u_{\varepsilon}\right|^{2} \mathrm{~d} m_{\varepsilon} \leq C, \quad \int_{\Omega} a_{\varepsilon}\left|\nabla u_{\varepsilon}\right|^{2} \mathrm{~d} x \leq C, \quad \int_{\Omega}\left|\nabla u_{\varepsilon}\right|^{2} \mathrm{~d} x \leq C,
$$

that is (3.14) and (3.17). By repeating the argument of the proof of Proposition 3.3, we infer the convergences (3.16), (3.18), (3.19). Assuming that $\gamma<+\infty$, we multiply (2.17) by the test function (4.2) (now with $\varphi, \psi$ chosen in $\mathcal{D}(\Omega))$, and after integration by parts we get

$$
\int_{\Omega} a_{\varepsilon} \nabla u_{\varepsilon} \cdot \nabla \Phi_{\varepsilon} \mathrm{d} x=\int_{\Omega} \rho_{\varepsilon} f \Phi_{\varepsilon} \mathrm{d} x .
$$

By the weak-star convergence in $\mathcal{M}(\bar{\Omega})$ of the sequence $\left(\rho_{\varepsilon} \Phi_{\varepsilon}\right)$ to the function $\rho_{0} \varphi+\bar{\rho}_{1}(\varphi+\psi)$ (see (4.14)), we have

$$
\lim _{\varepsilon \rightarrow 0} \int_{\Omega} \rho_{\varepsilon} f \Phi_{\varepsilon} \mathrm{d} x=\int_{\Omega}\left(\rho_{0} \varphi+\bar{\rho}_{1}(\varphi+\psi)\right) f \mathrm{~d} x .
$$

Splitting the term of the left hand side of (4.34) like in (4.17) and repeating the same argument we get (4.18), (4.23). Passing to the limit as $\varepsilon \rightarrow 0$ in (4.34), thanks to (4.18), (4.23), (4.17) and (4.35) we obtain

$$
\int_{\Omega} \nabla u \nabla \varphi \mathrm{d} x+4 \pi \gamma \int_{\Omega}(v-u) \psi \mathrm{d} x=\int_{\Omega}\left(\rho_{0} \varphi+\bar{\rho}_{1}(\varphi+\psi)\right) f \mathrm{~d} x
$$

and deduce from the arbitrary nature of $(\varphi, \psi)$ that $(u, v)$ is the unique solution in $H_{0}^{1}(\Omega) \times L^{2}(\Omega)$ of $(2.19)$ and $u$ is the unique solution in $H_{0}^{1}(\Omega)$ of $(2.18)$.

The case $\gamma=+\infty$ can be treated alike by fitting the argument of the proofs corresponding to evolution equations. 


\section{REFERENCES}

[1] M. Bellieud, Homogenization of evolution problems in a fiber reinforced structure. J. Convex Anal. 11 (2004) 363-385.

[2] M. Bellieud and G. Bouchitté, Homogenization of elliptic problems in a fiber reinforced structure. Non local effects. Ann. Scuola Norm. Sup. Cl. Sci. IV 26 (1998) 407-436.

[3] M. Bellieud and I. Gruais, Homogénéisation d'une structure élastique renforcée de fibres très rigides. Effets non locaux. $C$. $R$. Math., Problèmes mathématiques de la mécanique 337 (2003) 493-498.

[4] M. Bellieud and I. Gruais, Homogenization of an elastic material reinforced by very stiff or heavy fibers. Non local effects. Memory effects. J. Math. Pures Appl. 84 (2005) 55-96.

[5] H. Brezis, Analyse fonctionnelle. Masson, Paris (1983).

[6] G. Dal Maso, An introduction to Г-Convergence. Progress Nonlinear Differential Equations Appl., Birkhäuser, Boston (1993).

[7] E.Y. Khruslov, Homogenized models of composite media. Progress Nonlinear Differential Equations Appl., Birkhäuser (1991).

[8] J.L. Lions and E. Magenes, Problèmes aux limites non homogènes et applications. Dunod, Paris 1 (1968).

[9] U. Mosco, Composite media and asymptotic Dirichlet forms. J. Funct. Anal. 123 (1994) 368-421.

[10] G. Panasenko, Multicomponent homogenization of the vibration problem for incompressible media with heavy and rigid inclusions. C. R. Acad. Sci. Paris I 321 (1995) 1109-1114. 